\title{
The N-Aryl Aminocarbonyl ortho-Substituent Effect in \\ Cu-Catalyzed Aryl Amination and Its Application in the Synthesis of 5-Substituted 11-Oxo-dibenzodiazepines
}

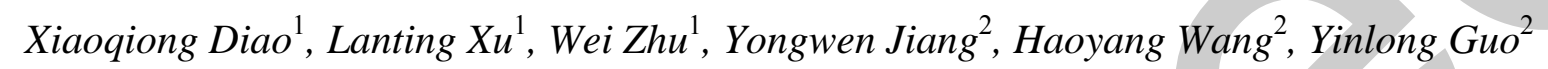
and Dawei $M a^{2} *$

${ }^{1}$ Department of Chemistry, Fudan University, Shanghai 200433, China

${ }^{2}$ State Key Laboratory of Bioorganic and Natural Products Chemistry, Shanghai Institute of Organic Chemistry, Chinese Academy of Sciences, 345 Lingling Lu, Shanghai 200032, China Madw@mail.sioc.ac.cn

\section{Supporting information}

\section{Table of contents}

Experimental- 


\section{Experimental}

\section{General procedure for CuCl-catalyzed formation of 11-oxo-dibenzodiazepines from}

dibromides. A Schlenk tube was charged with dibromides $(0.5 \mathrm{mmol}), \mathrm{CuCl}(5 \mathrm{mg}, 0.05$ mmol), 1,10-phenanthroline (9 mg, $0.05 \mathrm{mmol})$, and $\mathrm{K}_{3} \mathrm{PO}_{4}(318 \mathrm{mg}, 1.5 \mathrm{mmol})$. The tube was evacuated and backfilled with argon before amine $(0.55 \mathrm{mmol})$ and $1 \mathrm{~mL}$ DMF was added. The reaction mixture was stirred at room temperature for 3-12 h, and then stirred at 50 ${ }^{\circ} \mathrm{C}$ for 12-24 h. After the reaction mixture was diluted with EtOAc and washed with water and brine, the solution was dried over $\mathrm{Na}_{2} \mathrm{SO}_{4}$, and concentrated in vacuo. The residue was purified by column chromatography on silica gel (eluting with 50:1 petroleum ether/ethyl acetate) to provide the desired product.

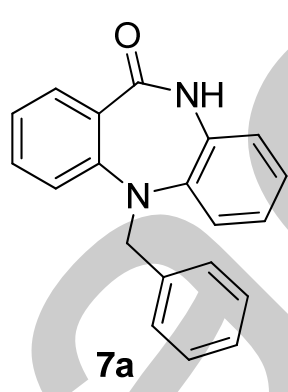

5-Benzyl-5H-dibenzo[b,e][1,4]diazepin-11(10H)-one (7a). $135 \mathrm{mg}$, Yield: 90\%. ${ }^{1} \mathrm{H}$ NMR $\left(400 \mathrm{MHz} \mathrm{CDCl}_{3}\right) \delta 8.93(\mathrm{~s}, 1 \mathrm{H}), 8.14(\mathrm{dd}, J=8.0,1.2 \mathrm{~Hz}, 1 \mathrm{H}), 7.71-7.69(\mathrm{~m}, 1 \mathrm{H}), 7.60-7.57$ (m, 1H), $7.43(\mathrm{~d}, J=7.5 \mathrm{~Hz}, 2 \mathrm{H}), 7.38-7.26(\mathrm{~m}, 6 \mathrm{H}), 6.77-6.72(\mathrm{~m}, 2 \mathrm{H}), 4.66(\mathrm{~d}, J=6.0 \mathrm{~Hz}$, $2 \mathrm{H}) .{ }^{13} \mathrm{C} \mathrm{NMR}\left(100 \mathrm{MHz}, \mathrm{CDCl}_{3}\right) \delta 163.51,149.34,148.49,141.93,139.45,132.99,129.20$, 128.87 (2C), 127.24, 127.09 (2C), 124.89, 124.48, 119.50, 115.68, 111.75, 110.45, 108.53, 47.22. ESI-HRMS calcd. for $\mathrm{C}_{20} \mathrm{H}_{17} \mathrm{~N}_{2} \mathrm{O}(\mathrm{M}+\mathrm{H})^{+}$requires $\mathrm{m} / \mathrm{z}$ 301.1341, found $\mathrm{m} / \mathrm{z}$ 301.1317 .

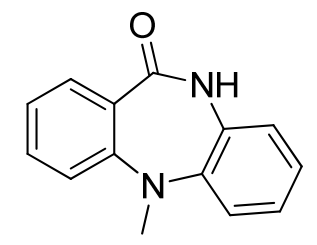

$7 b$

5-Methyl-5H-dibenzo[b,e][1,4]diazepin-11(10H)-one (7b). $82 \mathrm{mg}$, Yield: 73\%. ${ }^{1} \mathrm{H}$ NMR $\left(400 \mathrm{MHz}, \mathrm{CDCl}_{3}\right) \delta 8.25(\mathrm{~s}, 1 \mathrm{H}), 8.10(\mathrm{~d}, J=8.0 \mathrm{~Hz}, 1 \mathrm{H}), 7.71-7.69(\mathrm{~m}, 1 \mathrm{H}), 7.55-7.54(\mathrm{~m}$, 
1H), 7.39 (t, $J=7.8 \mathrm{~Hz}, 1 \mathrm{H}), 7.33-7.30(\mathrm{~m}, 2 \mathrm{H}), 6.78(\mathrm{~d}, J=8.8 \mathrm{~Hz}, 1 \mathrm{H}), 6.74$ (t, $J=7.6 \mathrm{~Hz}$, 1H), $3.06(\mathrm{~s}, 3 \mathrm{H}) ;{ }^{13} \mathrm{C}$ NMR (100 MHz, $\left.\mathrm{CDCl}_{3}\right) \delta 163.43,149.38,149.19,141.83,132.94$, 129.01, 124.66, 124.31, 119.22, 115.05, 110.56, 110.26, 108.06, 29.86. ESI-HRMS calcd. for $\mathrm{C}_{14} \mathrm{H}_{13} \mathrm{~N}_{2} \mathrm{O}(\mathrm{M}+\mathrm{H})^{+}$requires $\mathrm{m} / \mathrm{z} 225.1028$, found $\mathrm{m} / \mathrm{z} 225.1038$.

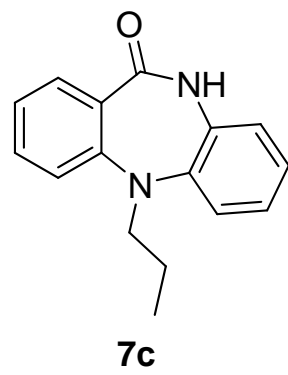

5-Propyl-5H-dibenzo[b,e][1,4]diazepin-11(10H)-one (7c). $92 \mathrm{mg}$, Yield: $73 \% .{ }^{1} \mathrm{H}$ NMR (400 MHz, $\left.\mathrm{CDCl}_{3}\right) \delta 8.39(\mathrm{~s}, 1 \mathrm{H}), 8.10$ (d, J=7.2 Hz, 1H), 7.71-7.69 (m, 1H), 7.57-7.54 (m, 1H), 7.38-7.31 (m, 3H), $6.80(\mathrm{~d}, J=8.4 \mathrm{~Hz}, 1 \mathrm{H}), 6.72(\mathrm{t}, J=7.3 \mathrm{~Hz}, 1 \mathrm{H}), 3.32(\mathrm{t}, J=6.4 \mathrm{~Hz}$, $2 \mathrm{H}), 1.86-1.80(\mathrm{~m}, 2 \mathrm{H}), 1.10(\mathrm{t}, J=7.4 \mathrm{~Hz}, 3 \mathrm{H}) .{ }^{13} \mathrm{C} \mathrm{NMR}\left(100 \mathrm{MHz}, \mathrm{CDCl}_{3}\right) \delta 163.49$, $149.18,148.66,141.91,132.86,129.12,124.62,124.26,119.30,114.85,111.01,110.24$, 107.89, 45.00, 22.58, 11.95. ESI-HRMS calcd. for $\mathrm{C}_{16} \mathrm{H}_{17} \mathrm{~N}_{2} \mathrm{O}(\mathrm{M}+\mathrm{H})^{+}$requires $\mathrm{m} / \mathrm{z}$ 253.1335 , found $\mathrm{m} / \mathrm{z} 253.1334$.

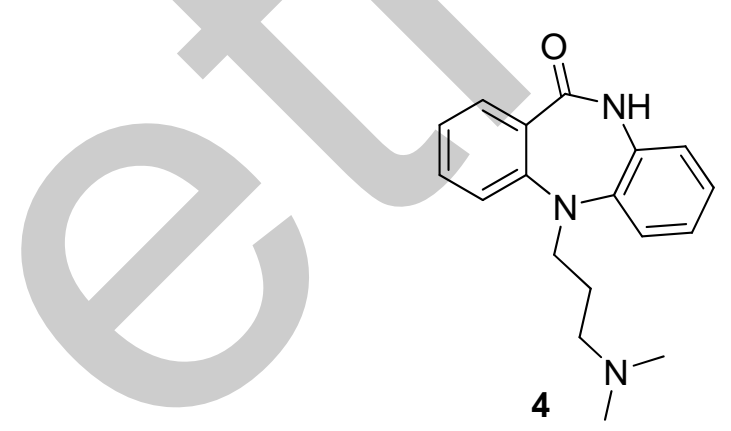

5-(3-(Dimethylamino)propyl)-5H-dibenzo[b,e][1,4]diazepin-11(10H)-one (4). $118 \mathrm{mg}$, Yield: $80 \% .{ }^{1} \mathrm{H}$ NMR (400 MHz, $\left.\mathrm{CDCl}_{3}\right) \delta 8.39(\mathrm{~s}, 1 \mathrm{H}), 8.10(\mathrm{~d}, J=8.0 \mathrm{~Hz}, 1 \mathrm{H}), 7.70-7.68$ (m, 1H), 7.56-7.54 (m, 1H), 7.37-7.31 (m, 3H), $6.81(\mathrm{~d}, J=8.4 \mathrm{~Hz}, 1 \mathrm{H}), 6.73(\mathrm{t}, J=7.5 \mathrm{~Hz}$, 1H), $3.44-3.39$ (m, 2H), 2.57 (t, $J=7.4 \mathrm{~Hz}, 2 \mathrm{H}), 2.35(\mathrm{~s}, 6 \mathrm{H}), 2.04-1.98(\mathrm{~m}, 2 \mathrm{H}) ;{ }^{13} \mathrm{C} \mathrm{NMR}$ $\left(100 \mathrm{MHz}, \mathrm{CDCl}_{3}\right) \delta 163.45,149.19,148.56,141.87,132.90,129.12,124.65,124.30,119.26$, 115.00, 111.05, 110.27, 108.01, 57.53, 45.73 (2C), 41.21, 27.39. ESI-HRMS calcd. for $\mathrm{C}_{18} \mathrm{H}_{22} \mathrm{~N}_{3} \mathrm{O}(\mathrm{M}+\mathrm{H})^{+}$requires $\mathrm{m} / \mathrm{z}$ 296.1757; found $\mathrm{m} / \mathrm{z} 296.1785$. 


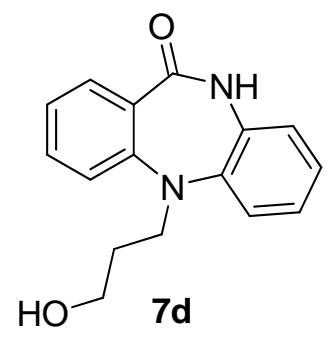

5-(3-Hydroxypropyl)-5H-dibenzo[b,e][1,4]diazepin-11(10H)-one (7d). $102 \mathrm{mg}$, Yield: 76\%. ${ }^{1} \mathrm{H}$ NMR (400 MHz, $\left.\mathrm{CDCl}_{3}\right) \delta 8.37$ (s, $\left.1 \mathrm{H}\right), 8.11(\mathrm{dd}, J=8.0,1.2 \mathrm{~Hz}, 1 \mathrm{H}), 7.71-7.69$ (m, 1H), 7.67-7.54 (m, 1H), 7.39-7.31 (m, 3H), $6.84(\mathrm{~d}, J=8.4 \mathrm{~Hz}, 1 \mathrm{H}), 6.74(\mathrm{t}, J=7.4 \mathrm{~Hz}, 1 \mathrm{H})$, $3.89(\mathrm{t}, J=6.0 \mathrm{~Hz}, 2 \mathrm{H}), 3.50(\mathrm{t}, J=6.4 \mathrm{~Hz}, 2 \mathrm{H}), 2.10-2.03(\mathrm{~m}, 2 \mathrm{H}) ;{ }^{13} \mathrm{C} \mathrm{NMR}(100 \mathrm{MHz}$, $\left.\mathrm{CDCl}_{3}\right) \delta 163.32,149.10,148.43,141.70,132.84,129.09,124.64,124.25,119.23,115.18$, 111.00, 110.19, 108.16, 60.85, 39.96, 32.04. ESI-HRMS calcd. for $\mathrm{C}_{16} \mathrm{H}_{17} \mathrm{~N}_{2} \mathrm{O}_{2}(\mathrm{M}+\mathrm{H})^{+}$ requires $\mathrm{m} / \mathrm{z} 269.1290$, found $\mathrm{m} / \mathrm{z} 269.1289$.

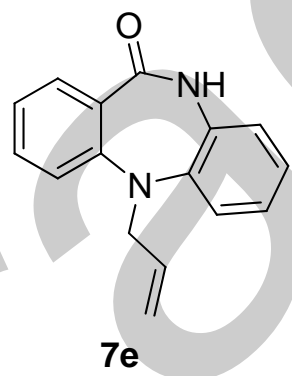

5-Allyl-5H-dibenzo[b,e][1,4]diazepin-11(10H)-one (7e). $102 \mathrm{mg}$, Yield: 82\%. ${ }^{1} \mathrm{H}$ NMR $\left(400 \mathrm{MHz} \mathrm{CDCl}_{3}\right) \delta 8.56(\mathrm{~s}, 1 \mathrm{H}), 8.12(\mathrm{dd}, J=8.0,1.6 \mathrm{~Hz}, 1 \mathrm{H}), 7.73-7.70(\mathrm{~m}, 1 \mathrm{H}), 7.58-7.56$ (m, 1H), 7.38-7.31 (m, 3H), 6.80-6.74 (m, 2H), 6.10-6.00 (m, 1H), 5.40 (dd, J=17.2, $1.2 \mathrm{~Hz}$, $1 \mathrm{H}), 5.24(\mathrm{dd}, J=10.4,1.6 \mathrm{~Hz}, 1 \mathrm{H}), 4.05(\mathrm{t}, J=5.2 \mathrm{~Hz}, 2 \mathrm{H}) ;{ }^{13} \mathrm{C} \mathrm{NMR}\left(100 \mathrm{MHz}, \mathrm{CDCl}_{3}\right) \delta$ $163.42,149.24,148.40,141.86,135.01,132.82,129.11,124.74,124.35,119.36,115.97$, 115.39, 111.46, 110.31, 108.31, 45.58. ESI-HRMS calcd. for $\mathrm{C}_{16} \mathrm{H}_{15} \mathrm{~N}_{2} \mathrm{O}(\mathrm{M}+\mathrm{H})^{+}$requires $\mathrm{m} / \mathrm{z} 251.1184$, found $\mathrm{m} / \mathrm{z} 251.1175$. 


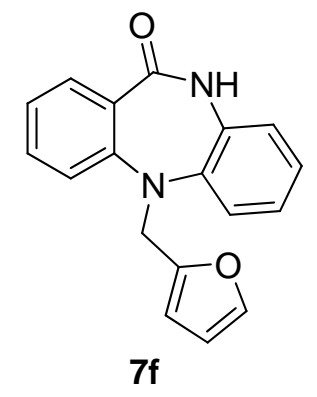

5-(Furan-2-ylmethyl)-5H-dibenzo[b,e][1,4]diazepin-11(10H)-one (7f). 99 mg, Yield: 68\%. ${ }^{1} \mathrm{H}$ NMR (400 MHz, $\left.\mathrm{CDCl}_{3}\right) \delta 8.80(\mathrm{~s}, 1 \mathrm{H}), 8.12(\mathrm{dd}, J=8.0,1.2 \mathrm{~Hz}, 1 \mathrm{H}), 7.72-7.70(\mathrm{~m}, 1 \mathrm{H})$, 7.57-7.55 (m, 1H), 7.40-7.31 (m, 4H), $6.86(\mathrm{~d}, J=8.4 \mathrm{~Hz}, 1 \mathrm{H}), 6.78(\mathrm{t}, J=7.5 \mathrm{~Hz}, 1 \mathrm{H})$, 6.35-6.34 (m, 1H), 6.31-6.30 (m, 1H), 4.60 (d, J = 5.6 Hz, 2H); ${ }^{13} \mathrm{C}$ NMR (100 MHz, CDCl $)$ $\delta 163.22,152.79,149.20,148.02,141.93,141.74,132.81,129.08,124.77,124.35,119.41$, 115.87, 111.34, 110.45, 110.29, 108.68, 106.81, 40.67; ESI-HRMS calcd. for $\mathrm{C}_{18} \mathrm{H}_{14} \mathrm{~N}_{2} \mathrm{O}_{2} \mathrm{Na}$ $(\mathrm{M}+\mathrm{Na})^{+}$requires $\mathrm{m} / \mathrm{z} 313.0953$; found $\mathrm{m} / \mathrm{z} 313.0948$.

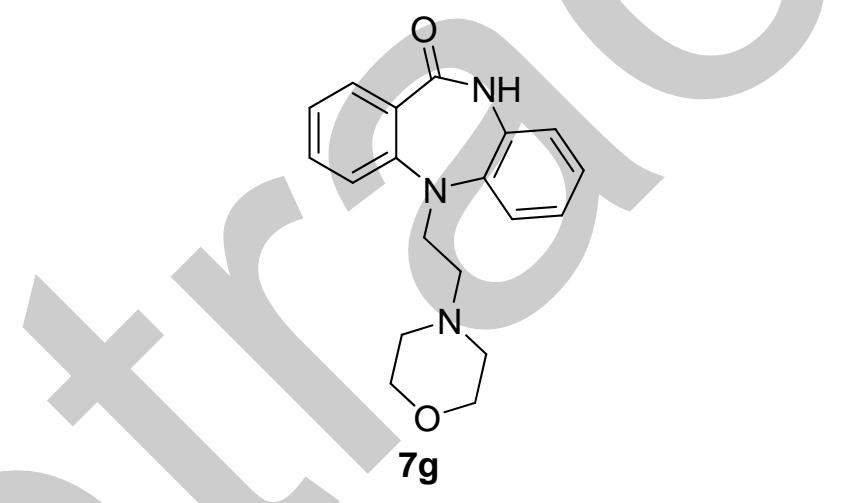

5-(2-Morpholinoethyl)-5H-dibenzo[b,e $][1,4]$ diazepin-11(10H)-one (7g). $121 \mathrm{mg}$, Yield: $75 \% .{ }^{1} \mathrm{H}$ NMR $\left(400 \mathrm{MHz}, \mathrm{CDCl}_{3}\right) \delta 8.62(\mathrm{~s}, 1 \mathrm{H}), 8.11(\mathrm{dd}, J=8.0,1.2 \mathrm{~Hz}, 1 \mathrm{H}), 7.72-7.69(\mathrm{~m}$, 1H), 7.57-7.55 (m, 1H), 7.37-7.32 (m, 3H), 6.80-6.73 (m, 2H), 3.83-3.81 (m, 4H), 3.46-3.42 $(\mathrm{m}, 2 \mathrm{H}), 2.80(\mathrm{t}, J=6.4 \mathrm{~Hz}, 2 \mathrm{H}), 2.60(\mathrm{~s}, 4 \mathrm{H}) ;{ }^{13} \mathrm{C} \mathrm{NMR}\left(100 \mathrm{MHz}, \mathrm{CDCl}_{3}\right) \delta 163.32,149.27$, $148.37,141.99,132.90,129.21,124.70,124.34,119.25,115.23,111.19,110.32,108.42$, 67.35 (2C), 56.95, 53.59 (2C), 40.10. ESI-HRMS calcd. for $\mathrm{C}_{19} \mathrm{H}_{22} \mathrm{~N}_{3} \mathrm{O}_{2}(\mathrm{M}+\mathrm{H})^{+}$requires $\mathrm{m} / \mathrm{z}$ 324.1707, found $\mathrm{m} / \mathrm{z} 324.1706$. 


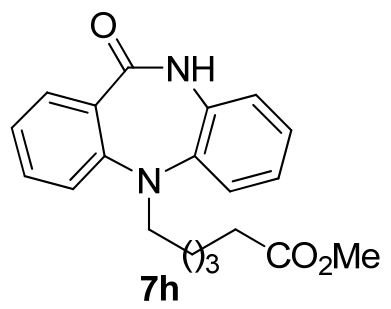

Methyl 6-(11-oxo-10,11-dihydro-5H-dibenzo[b,e][1,4]diazepin-5-yl)hexanoate (7h). 135 mg, Yield: 80\%. ${ }^{1} \mathrm{H}$ NMR (400 MHz, $\left.\mathrm{CDCl}_{3}\right) \delta 8.36(\mathrm{~s}, 1 \mathrm{H}), 8.11(\mathrm{~d}, J=8.0 \mathrm{~Hz}, 1 \mathrm{H})$, 7.73-7.71 (m, 1H), 7.56-7.55 (m, 1H), 7.38-7.30 (m, 3H), $6.78(\mathrm{~d}, J=8.5 \mathrm{~Hz}, 1 \mathrm{H}), 6.73(\mathrm{t}, J=$ $7.5 \mathrm{~Hz}, 1 \mathrm{H}), 3.67(\mathrm{~s}, 3 \mathrm{H}), 3.37-3.32(\mathrm{~m}, 2 \mathrm{H}), 2.38(\mathrm{t}, J=7.5 \mathrm{~Hz}, 2 \mathrm{H}), 1.87-1.72(\mathrm{~m}, 4 \mathrm{H})$, 1.60-1.52 (m, 2H); ${ }^{13} \mathrm{C}$ NMR (100 MHz, $\left.\mathrm{CDCl}_{3}\right) \delta 174.31,163.53,149.26,148.63,141.95$, $132.98,129.22,124.75,124.39,119.42,115.05,111.05,110.35,108.04,51.74,43.05,34.25$, 29.06, 27.02, 24.96; ESI-HRMS calcd. for $\mathrm{C}_{20} \mathrm{H}_{23} \mathrm{~N}_{2} \mathrm{O}_{3}(\mathrm{M}+\mathrm{H})^{+}$requires $\mathrm{m} / \mathrm{z}$ 339.1709; found $\mathrm{m} / \mathrm{z} 339.1695$.

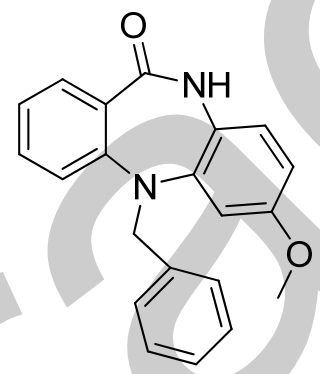

$7 \mathbf{i}$

5-Benzyl-7-methoxy-5H-dibenzo[b,e][1,4]diazepin-11(10H)-one (7i). $125 \mathrm{mg}$, Yield: 76\%. ${ }^{1} \mathrm{H}$ NMR $\left(400 \mathrm{MHz}, \mathrm{CDCl}_{3}\right) \delta 8.83(\mathrm{~s}, 1 \mathrm{H}), 8.08(\mathrm{dd}, J=8.0,1.2 \mathrm{~Hz}, 1 \mathrm{H}), 7.56(\mathrm{~d}, J=8.7 \mathrm{~Hz}$, 1H), $7.42(\mathrm{~d}, J=7.3 \mathrm{~Hz}, 2 \mathrm{H}), 7.35(\mathrm{t}, J=7.5 \mathrm{~Hz}, 2 \mathrm{H}), 7.28-7.24(\mathrm{~m}, 2 \mathrm{H}), 7.12(\mathrm{~d}, J=2.0 \mathrm{~Hz}$, 1H), $6.93(\mathrm{dd}, J=8.4,2.4 \mathrm{~Hz}, 1 \mathrm{H}), 6.75-6.69(\mathrm{~m}, 2 \mathrm{H}), 4.64(\mathrm{~d}, J=5.8 \mathrm{~Hz}, 2 \mathrm{H}), 3.89$ (s, 3H); ${ }^{13} \mathrm{C}$ NMR $\left(100 \mathrm{MHz} \mathrm{CDCl}_{3}\right) \delta 162.71,158.18,150.24,148.00,139.51,135.67,132.51$, 128.84 (2C), 128.79, 127.20, 127.09 (2C), 119.48, 115.64, 112.46, 111.64, 108.86, 95.50, 56.20, 47.22; ESI-HRMS calcd. for $\mathrm{C}_{21} \mathrm{H}_{18} \mathrm{~N}_{2} \mathrm{NaO}_{2}(\mathrm{M}+\mathrm{Na})^{+}$requires $\mathrm{m} / \mathrm{z}$ 353.1266, found $\mathrm{m} / \mathrm{z} 353.1251$.

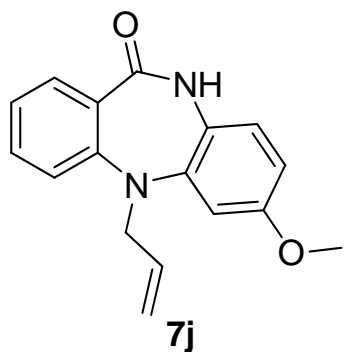


5-Allyl-7-methoxy-5H-dibenzo[b,e][1,4]diazepin-11(10H)-one (7j). $112 \mathrm{mg}$, Yield: 80\%. ${ }^{1} \mathrm{H}$ NMR (400 MHz, $\left.\mathrm{CDCl}_{3}\right) \delta 8.47(\mathrm{~s}, 1 \mathrm{H}), 8.07(\mathrm{~d}, J=7.2 \mathrm{~Hz}, 1 \mathrm{H}), 7.58(\mathrm{~d}, J=8.8 \mathrm{~Hz}, 1 \mathrm{H})$, $7.33(\mathrm{t}, J=7.4 \mathrm{~Hz}, 1 \mathrm{H}), 7.11(\mathrm{~d}, J=2.0 \mathrm{~Hz}, 1 \mathrm{H}), 6.93(\mathrm{dd}, J=8.8,2.0 \mathrm{~Hz}, 1 \mathrm{H}), 6.78-6.73(\mathrm{~m}$, 2H), 6.09-6.02 (m, 1H), 5.39 (dd, $J=17.2,0.9 \mathrm{~Hz}, 1 \mathrm{H}), 5.24(\mathrm{dd}, J=10.4,0.8 \mathrm{~Hz}, 1 \mathrm{H}), 4.03$ (s, 2H), $3.88(\mathrm{~s}, 3 \mathrm{H}) ;{ }^{13} \mathrm{C} \mathrm{NMR}\left(100 \mathrm{MHz}, \mathrm{CDCl}_{3}\right) \delta\left(101 \mathrm{MHz}, \mathrm{CDCl}_{3}\right) 162.60,158.06$, $150.10,147.44,135.58,135.05,132.34,128.68,119.32,115.93,115.35,112.31,111.33$, 108.61, 95.40, 56.06, 45.57; ESI-HRMS calcd. for $\mathrm{C}_{17} \mathrm{H}_{17} \mathrm{~N}_{2} \mathrm{O}_{2}(\mathrm{M}+\mathrm{H})^{+}$requires $\mathrm{m} / \mathrm{z}$ 281.1290 , found $\mathrm{m} / \mathrm{z} 281.1288$.

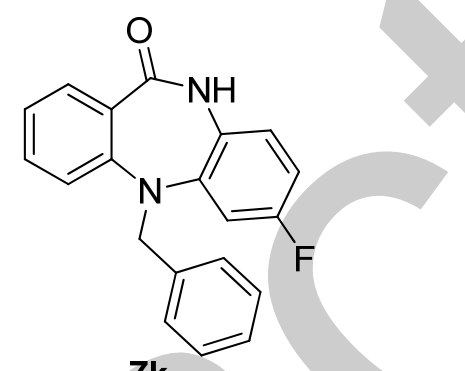

$7 \mathrm{k}$

5-Benzyl-7-fluoro-5H-dibenzo[b,e][1,4]diazepin-11(10H)-one (7k). $111 \mathrm{mg}$, Yield: 70\%. ${ }^{1} \mathrm{H}$ NMR $\left(400 \mathrm{MHz}, \mathrm{CDCl}_{3}\right) \delta 8.79(\mathrm{~s}, 1 \mathrm{H}), 8.09(\mathrm{~d}, J=8.0 \mathrm{~Hz}, 1 \mathrm{H}), 7.61(\mathrm{dd}, J=8.8,4.8 \mathrm{~Hz}$, 1H), $7.43(\mathrm{~d}, J=7.5 \mathrm{~Hz}, 2 \mathrm{H}), 7.37$ (t, $J=7.5 \mathrm{~Hz}, 2 \mathrm{H}), 7.31-7.27(\mathrm{~m}, 3 \mathrm{H}), 7.08$ (dt, $J=9.2$, $2.3 \mathrm{~Hz}, 1 \mathrm{H}), 6.77-6.72(\mathrm{~m}, 2 \mathrm{H}), 4.64(\mathrm{~d}, J=5.8 \mathrm{~Hz}, 2 \mathrm{H}) ;{ }^{13} \mathrm{C} \mathrm{NMR}\left(100 \mathrm{MHz}, \mathrm{CDCl}_{3}\right) \delta(100$ $\left.\mathrm{MHz}, \mathrm{CDCl}_{3}\right) 163.01$ (d, $\left.J=231.3 \mathrm{~Hz}\right), 159.44,149.35$ (d, $\left.J=14.8 \mathrm{~Hz}\right), 148.29,139.35$, 138.22, 133.08, 129.07, 128.88 (2C), 127.28, 127.10 (2C), 119.61 (d, $J=10.0 \mathrm{~Hz}), 115.75$, $112.25(\mathrm{~d}, J=24.6 \mathrm{~Hz}), 111.76,108.27,98.62(\mathrm{~d}, J=28.4 \mathrm{~Hz}), 47.23$; ESI-HRMS calcd. for $\mathrm{C}_{20} \mathrm{H}_{15} \mathrm{FN}_{2} \mathrm{NaO}(\mathrm{M}+\mathrm{Na})^{+}$requires $\mathrm{m} / \mathrm{z} 341.1066$, found $\mathrm{m} / \mathrm{z} 341.1059$.<smiles>C=CCN1c2ccccc2NC(=O)c2cc(OC)ccc21</smiles>

5-Allyl-2-methoxy-5H-dibenzo[b,e][1,4]diazepin-11(10H)-one (7l). $84 \mathrm{mg}$, Yield: $60 \% .{ }^{1} \mathrm{H}$ NMR $\left(400 \mathrm{MHz}, \mathrm{CDCl}_{3}\right) \delta 8.20(\mathrm{~s}, 1 \mathrm{H}), 7.73-7.71(\mathrm{~m}, 1 \mathrm{H}), 7.65(\mathrm{~d}, J=2.8 \mathrm{~Hz}, 1 \mathrm{H})$, 
7.58-7.56 (m, 1H), 7.34-7.32 (m, 2H), $7.03(\mathrm{dd}, J=9.1,2.8 \mathrm{~Hz}, 1 \mathrm{H}), 6.75(\mathrm{~d}, J=9.1 \mathrm{~Hz}, 1 \mathrm{H})$, 6.09-6.01 (m, 1H), 5.37 (d, $J=17.1 \mathrm{~Hz}, 1 \mathrm{H}), 5.22$ (d, $J=10.3 \mathrm{~Hz}, 1 \mathrm{H}), 4.01$ (s, 2H), 3.86 (s, $3 \mathrm{H}) ;{ }^{13} \mathrm{C} \mathrm{NMR}\left(100 \mathrm{MHz}, \mathrm{CDCl}_{3}\right) \delta 163.13,150.08,149.20,143.63,141.93,135.41,124.79$, 124.41, 121.28, 119.44, 115.84, 113.02, 112.18, 110.30, 108.07, 56.22, 46.00; ESI-HRMS calcd. for $\mathrm{C}_{17} \mathrm{H}_{17} \mathrm{~N}_{2} \mathrm{O}_{2}(\mathrm{M}+\mathrm{H})^{+}$requires $\mathrm{m} / \mathrm{z} 281.1285$, found $\mathrm{m} / \mathrm{z} 281.1285$.

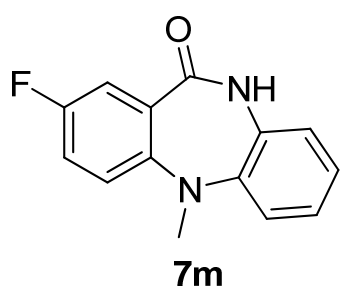

2-Fluoro-5-methyl-5H-dibenzo[b,e][1,4]diazepin-11(10H)-one (7m). $64 \mathrm{mg}$, Yield: 53\%. ${ }^{1} \mathrm{H}$ NMR $\left(400 \mathrm{MHz}, \mathrm{CDCl}_{3}\right) \delta 8.05(\mathrm{~s}, 1 \mathrm{H}), 7.79(\mathrm{dd}, J=9.6,2.8 \mathrm{~Hz}, 1 \mathrm{H}), 7.72-7.70(\mathrm{~m}, 1 \mathrm{H})$, 7.56-7.53 (m, 1H), 7.35-7.33 (m, 2H), $7.13(\mathrm{dt}, J=8.8,2.8 \mathrm{~Hz}, 1 \mathrm{H}), 6.68(\mathrm{dd}, J=9.2,4.4 \mathrm{~Hz}$, $1 \mathrm{H}), 3.03(\mathrm{~d}, J=5.0 \mathrm{~Hz}, 3 \mathrm{H}) ;{ }^{13} \mathrm{C} \mathrm{NMR}\left(100 \mathrm{MHz}, \mathrm{CDCl}_{3}\right) \delta 153.82(\mathrm{~d}, J=233.1 \mathrm{~Hz})$, 149.28, 146.29, 141.82, 134.53, 125.17, 124.65, 120.35 (d, $J=22.9 \mathrm{~Hz}), 119.55,114.53(\mathrm{~d}, J$ $=24.5 \mathrm{~Hz}), 111.611(\mathrm{~d}, J=7.3 \mathrm{~Hz}), 110.51,107.88(\mathrm{~d}, J=7.7 \mathrm{~Hz}), 30.33$; ESI-HRMS calcd. for $\mathrm{C}_{14} \mathrm{H}_{12} \mathrm{FN}_{2} \mathrm{O}(\mathrm{M}+\mathrm{H})^{+}$requires $\mathrm{m} / \mathrm{z}$ 243.0934; found $\mathrm{m} / \mathrm{z} 243.0926$.

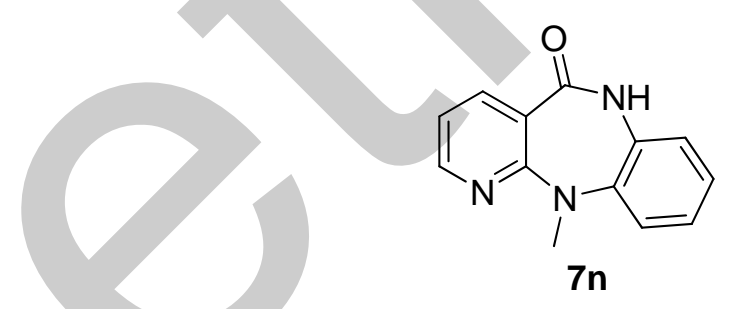

11-Methyl-6,11-dihydro-5H-benzo[b]pyrido[2,3-e][1,4]diazepin-5-one (7n). $62 \mathrm{mg}$, Yield: $55 \% .{ }^{1} \mathrm{H}$ NMR $\left(400 \mathrm{MHz}, \mathrm{CDCl}_{3}\right) \delta 8.59(\mathrm{~s}, 1 \mathrm{H}), 8.32(\mathrm{dd}, J=4.8,1.7 \mathrm{~Hz}, 1 \mathrm{H}), 8.26(\mathrm{dd}, J=$ 7.7, $1.8 \mathrm{~Hz}, 1 \mathrm{H}), 7.72-7.70(\mathrm{~m}, 1 \mathrm{H}), 7.55-7.53(\mathrm{~m}, 1 \mathrm{H}), 7.36-7.31(\mathrm{~m}, 2 \mathrm{H}), 6.64(\mathrm{dd}, J=7.6$, $4.8 \mathrm{~Hz}, 1 \mathrm{H}), 3.20(\mathrm{~d}, J=4.8 \mathrm{~Hz}, 3 \mathrm{H}) ;{ }^{13} \mathrm{C} \mathrm{NMR}\left(100 \mathrm{MHz}, \mathrm{CDCl}_{3}\right) \delta 161.96,157.12,151.84$, 149.69, 141.58, 136.82, 125.27, 124.75, 119.66, 111.23, 110.50, 104.54, 28.37; ESI-HRMS calcd. for $\mathrm{C}_{13} \mathrm{H}_{12} \mathrm{~N}_{3} \mathrm{O}(\mathrm{M}+\mathrm{H})^{+}$requires $\mathrm{m} / \mathrm{z}$ 226.0980, found $\mathrm{m} / \mathrm{z} 226.0960$. 


\section{General procedure for $\mathrm{CuCl}$-catalyzed coupling of 2-halo- $N$-phenyl-benzamides with}

N-nucleophiles. A Schlenk tube was charged with dibromides (1 mmol), $\mathrm{CuCl}(1 \mathrm{mg}, 0.01$ mmol) and $\mathrm{K}_{3} \mathrm{PO}_{4}(424 \mathrm{mg}, 2 \mathrm{mmol})$. The tube was evacuated and backfilled with argon before $\mathrm{N}$-nucleophile $(2 \mathrm{mmol}$ ) and $2 \mathrm{~mL}$ DMF was added. The reaction mixture was stirred at room temperature for 10-36 h. After EtOAc was added, and the organic layer was washed with water and brine, dried over $\mathrm{Na}_{2} \mathrm{SO}_{4}$ and concentrated in vacuo. The residue was purified by column chromatography on silica gel (eluting with 10:1 petroleum ether/ethyl acetate) to provide the desired product.

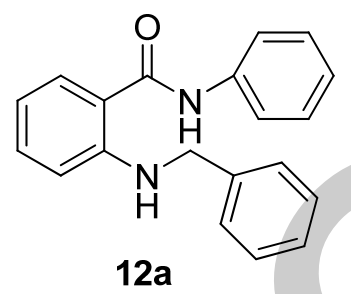

2-(Benzylamino)- $N$-phenylbenzamide (12a). $281 \mathrm{mg}$, Yield: $93 \%$. ${ }^{1} \mathrm{H}$ NMR (400 MHz, $\left.\mathrm{CDCl}_{3}\right) \delta 7.95(\mathrm{~s}, 1 \mathrm{H}), 7.82(\mathrm{~s}, 1 \mathrm{H}), 7.56(\mathrm{dd}, J=8.6,1.0 \mathrm{~Hz}, 2 \mathrm{H}), 7.51(\mathrm{dd}, J=7.8,1.5 \mathrm{~Hz}$, $1 \mathrm{H}), 7.39-7.25(\mathrm{~m}, 8 \mathrm{H}), 7.17-7.14(\mathrm{~m}, 1 \mathrm{H}), 6.70-6.63(\mathrm{~m}, 2 \mathrm{H}), 4.42(\mathrm{~d}, J=4.9 \mathrm{~Hz}, 2 \mathrm{H}) .{ }^{13} \mathrm{C}$ NMR (100 MHz, $\left.\mathrm{CDCl}_{3}\right) \delta 168.30,149.90,139.04,137.94,133.39,129.18$ (2C), $128.76(2 \mathrm{C})$, 127.50, 127.27 (2C), 127.21, 124.64, 120.80 (2C), 115.48, 115.30, 112.51, 47.28; ESI-HRMS calcd. for $\mathrm{C}_{20} \mathrm{H}_{19} \mathrm{~N}_{2} \mathrm{O}(\mathrm{M}+\mathrm{H})^{+}$requires $m / z$ 303.1497, found $m / z$ 303.1498.

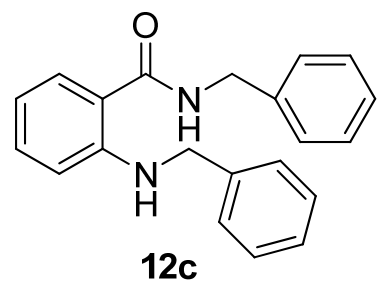

N-Benzyl-2-(benzylamino)benzamide (12c). $265 \mathrm{mg}$, Yield: 84\%. ${ }^{1} \mathrm{H}$ NMR $(400 \mathrm{MHz}$, $\left.\mathrm{CDCl}_{3}\right) \delta 8.13(\mathrm{~s}, 1 \mathrm{H}), 7.39-7.29(\mathrm{~m}, 10 \mathrm{H}), 7.27-7.19(\mathrm{~m}, 2 \mathrm{H}), 6.64(\mathrm{dd}, J=8.4,0.7 \mathrm{~Hz}, 1 \mathrm{H})$, 6.59-6.55 (m, 1H), $6.35(\mathrm{~s}, 1 \mathrm{H}), 4.60$ (d, $J=5.6 \mathrm{~Hz}, 2 \mathrm{H}), 4.42(\mathrm{~s}, 2 \mathrm{H}) ;{ }^{13} \mathrm{C} \mathrm{NMR}(100 \mathrm{MHz}$, $\left.\mathrm{CDCl}_{3}\right) \delta 169.72,149.62,139.18,138.35,133.01,128.89$ (2C), $128.71(2 \mathrm{C}), 127.89$ (2C), 127.68, 127.31, 127.21 (2C), 127.11, 115.17, 112.43, 47.30, 43.85; ESI-HRMS calcd. for $\mathrm{C}_{20} \mathrm{H}_{21} \mathrm{~N}_{2} \mathrm{O}(\mathrm{M}+\mathrm{H})^{+}$requires $\mathrm{m} / \mathrm{z} 317.1654$, found $\mathrm{m} / \mathrm{z} 317.1622$. 


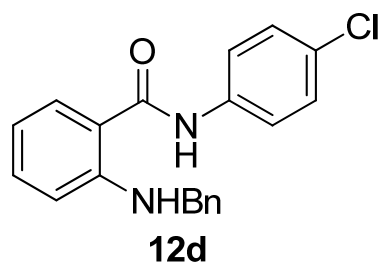

2-(Benzylamino)-N-(4-chlorophenyl)benzamide (12d). $286 \mathrm{mg}$, Yield: 85\%. ${ }^{1} \mathrm{H}$ NMR (400 $\left.\mathrm{MHz}, \mathrm{CDCl}_{3}\right) \delta 7.89(\mathrm{~s}, 1 \mathrm{H}), 7.80(\mathrm{~s}, 1 \mathrm{H}), 7.44-7.47(\mathrm{~m}, 3 \mathrm{H}), 7.40-7.14(\mathrm{~m}, 8 \mathrm{H}), 6.66(\mathrm{~d}, J=$ $8.4 \mathrm{~Hz}, 1 \mathrm{H}), 6.61(\mathrm{t}, J=7.6 \mathrm{~Hz}, 1 \mathrm{H}), 4.38(\mathrm{~d}, J=5.6 \mathrm{~Hz}, 2 \mathrm{H}) ;{ }^{13} \mathrm{C} \mathrm{NMR}\left(100 \mathrm{MHz}, \mathrm{CDCl}_{3}\right) \delta$ $168.22,149.85,138.85,136.46,133.53,129.48,129.06,128.72,127.47,127.20,121.98$, 115.29, 114.99, 112.51, 47.24. ESI-HRMS calcd. for $\mathrm{C}_{20} \mathrm{H}_{17} \mathrm{ClN}_{2} \mathrm{O}(\mathrm{M}+\mathrm{H})^{+}$requires $\mathrm{m} / \mathrm{z}$ 337.1108, found $\mathrm{m} / \mathrm{z} 337.1081$.<smiles>COc1ccc(NC(=O)c2ccccc2NCc2ccccc2)cc1</smiles>

$12 \mathrm{e}$

2-(Benzylamino)-N-(4-methoxyphenyl)benzamide (12e). $133 \mathrm{mg}$, Yield: 40\%. ${ }^{1} \mathrm{H}$ NMR $\left(400 \mathrm{MHz}, \mathrm{CDCl}_{3}\right) \delta 7.93(\mathrm{~s}, 1 \mathrm{H}), 7.72(\mathrm{~s}, 1 \mathrm{H}), 7.46(\mathrm{~d}, J=8.0 \mathrm{~Hz}, 1 \mathrm{H}), 7.41(\mathrm{~d}, J=8.8 \mathrm{~Hz}$, 2H), 7.37-7.18 (m, 6H), $6.87(\mathrm{~d}, J=9.2 \mathrm{~Hz}, 2 \mathrm{H}), 6.59-6.65(\mathrm{~m}, 2 \mathrm{H}), 4.38(\mathrm{~d}, J=5.2 \mathrm{~Hz}, 2 \mathrm{H})$, $3.78(\mathrm{~s}, 3 \mathrm{H}) ;{ }^{13} \mathrm{C}$ NMR $\left(100 \mathrm{MHz}, \mathrm{CDCl}_{3}\right) \delta 168.25,156.72,149.76,139.04,133.18,130.82$, $128.69,127.43,127.24,127.13,122.87,115.48,115.20,114.29,112.36,55.58,47.25$. ESI-HRMS calcd. for $\mathrm{C}_{21} \mathrm{H}_{20} \mathrm{~N}_{2} \mathrm{O}_{2}(\mathrm{M}+\mathrm{H})^{+}$requires $\mathrm{m} / \mathrm{z} 333.1603$, found $\mathrm{m} / \mathrm{z} 333.1597$.

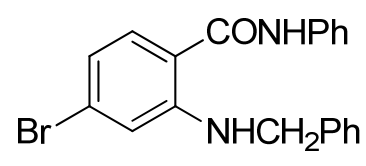
15

2-(Benzylamino)-4-bromo-N-phenylbenzamide (15). 35 mg, Yield: 81\% (2,4-dibromo-Nphenylbenzamide: $40 \mathrm{mg}, 0.11 \mathrm{mmol}) .{ }^{1} \mathrm{H} \mathrm{NMR}\left(400 \mathrm{MHz}, \mathrm{CDCl}_{3}\right) \delta 8.00(\mathrm{t}, J=4.8 \mathrm{~Hz}, 1 \mathrm{H})$, $7.69(\mathrm{~s}, 1 \mathrm{H}), 7.50(\mathrm{~d}, J=8.0 \mathrm{~Hz}, 2 \mathrm{H}), 7.30-7.36(\mathrm{~m}, 7 \mathrm{H}), 7.24-7.27(\mathrm{~m}, 1 \mathrm{H}), 7.14(\mathrm{t}, J=7.6$ $\mathrm{Hz}, 1 \mathrm{H}), 6.83(\mathrm{~d}, J=1.6 \mathrm{~Hz}, 1 \mathrm{H}), 6.75(\mathrm{dd}, J=1.6,8.0 \mathrm{~Hz}, 1 \mathrm{H}), 4.35(\mathrm{~d}, J=5.6 \mathrm{~Hz}, 2 \mathrm{H}) ;{ }^{13} \mathrm{C}$ NMR (100 MHz, $\left.\mathrm{CDCl}_{3}\right) \delta 167.57,150.70,138.07,137.55,129.15$ (2C), 128.80 (2C), 128.59, 
<smiles>C1CC(C2CC2)C1</smiles> 


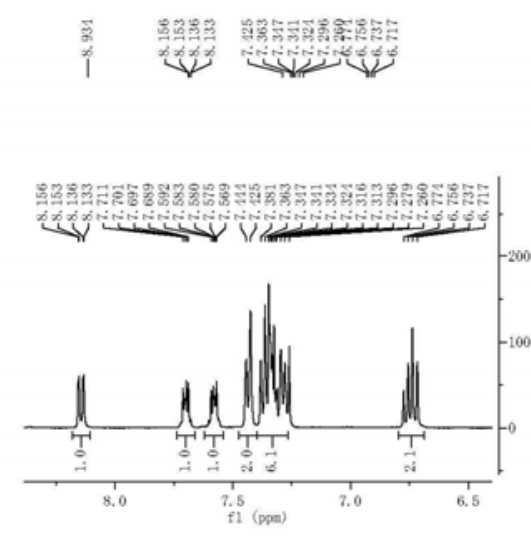

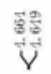
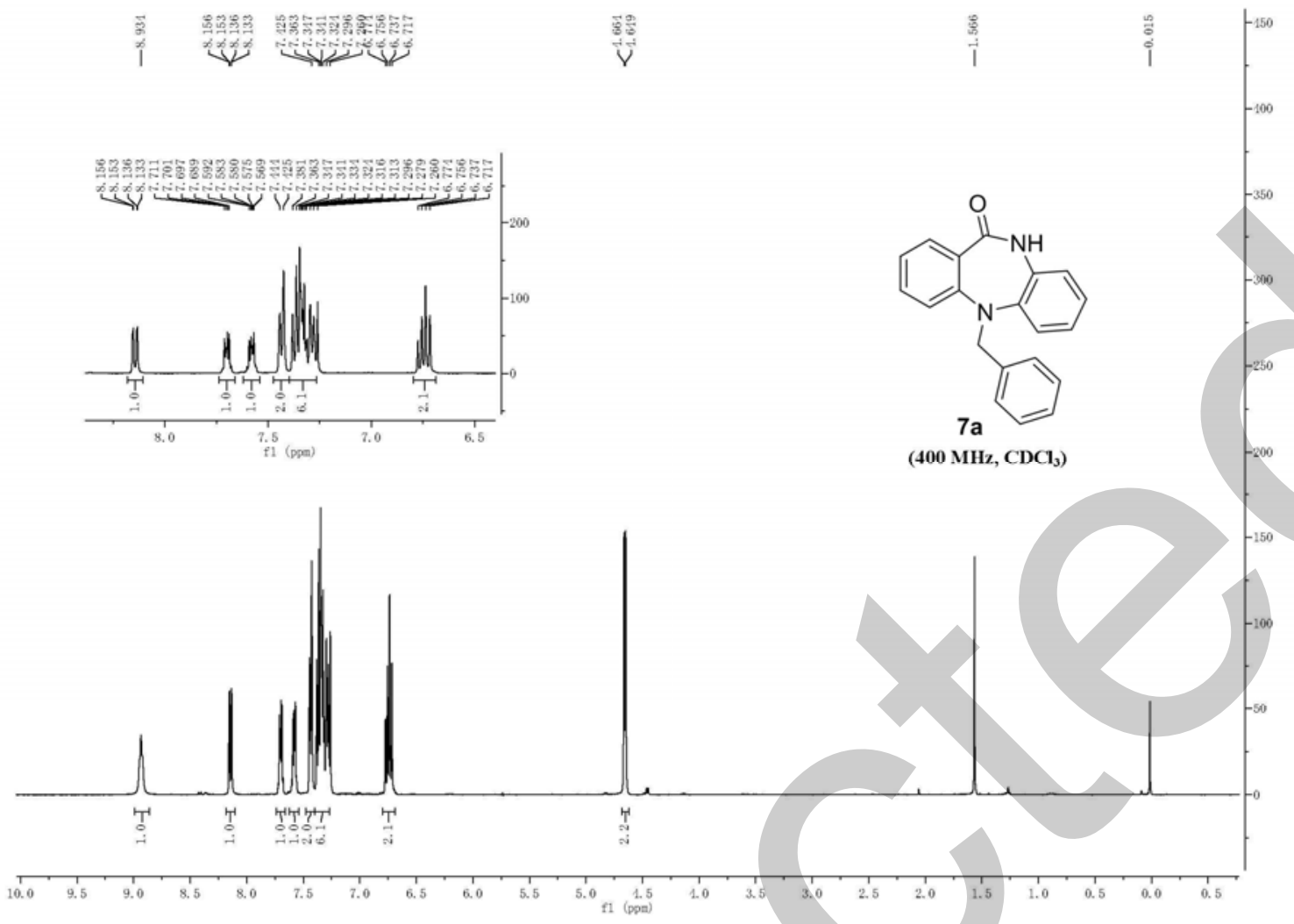

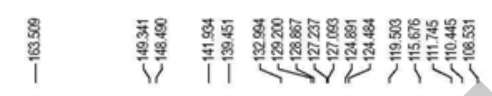
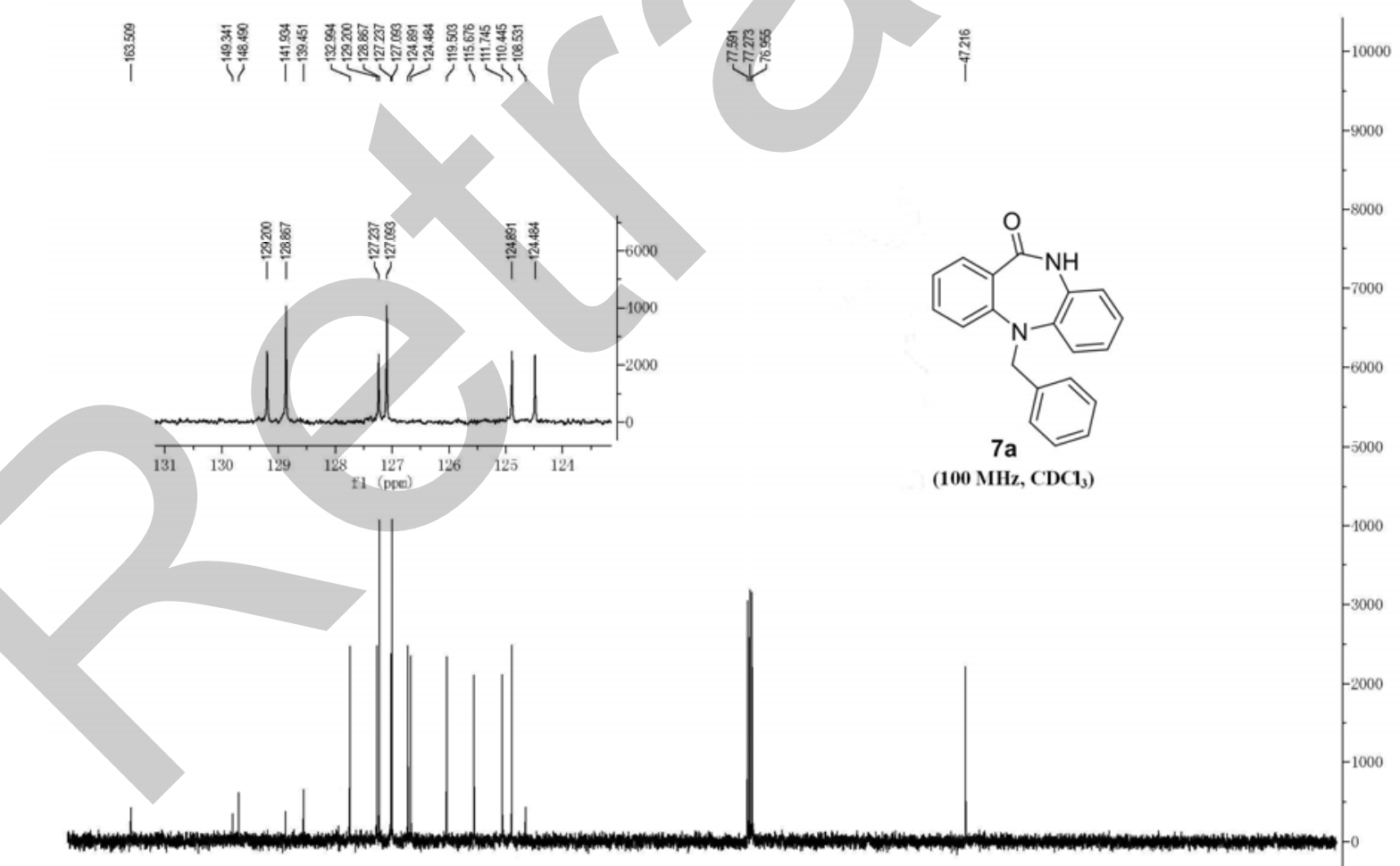
(100 $\left.\mathrm{MHz}_{2}, \mathrm{CDCl}_{3}\right)$

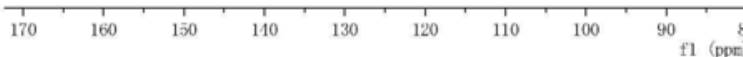




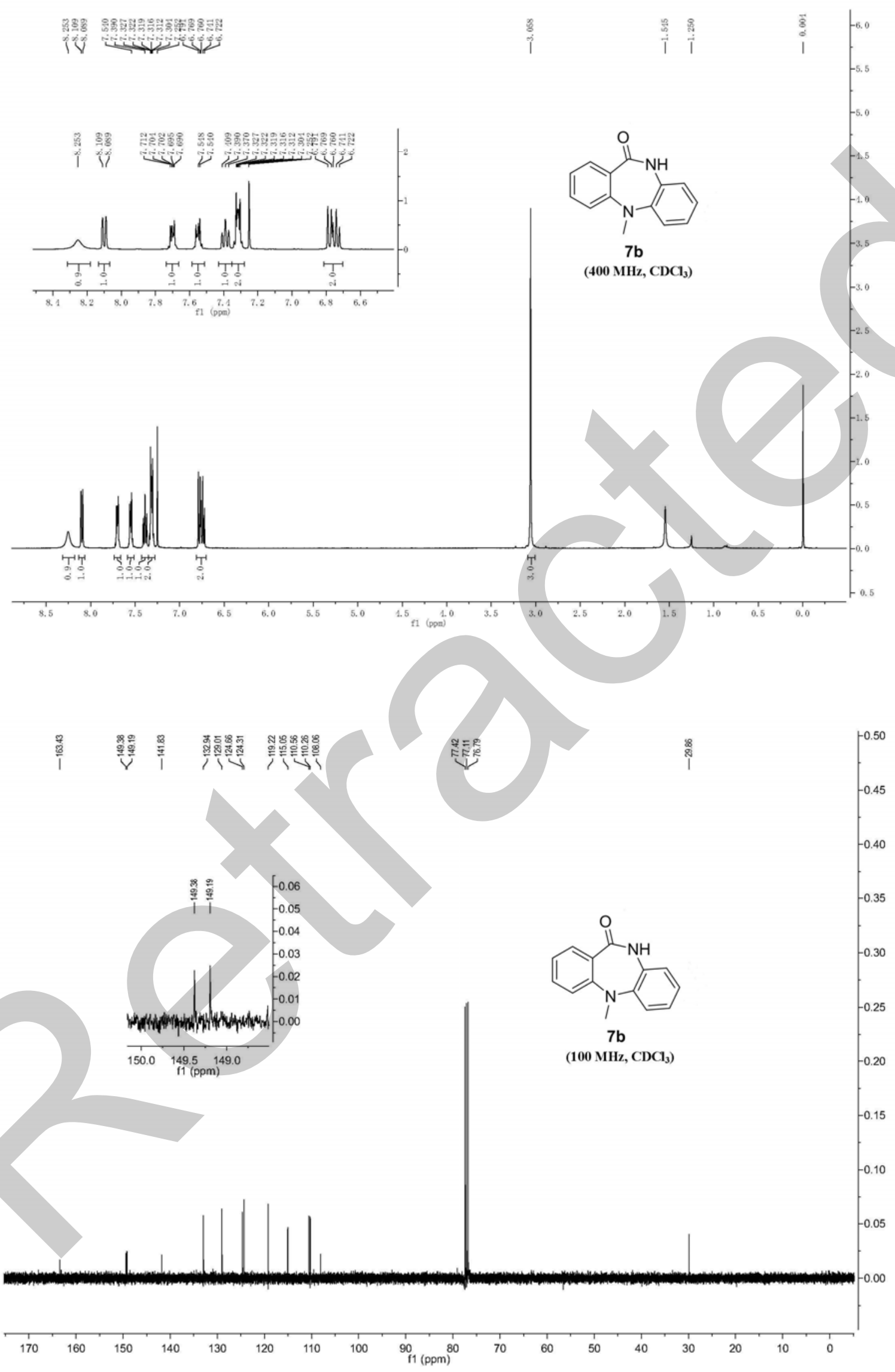




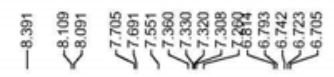

รัตู้
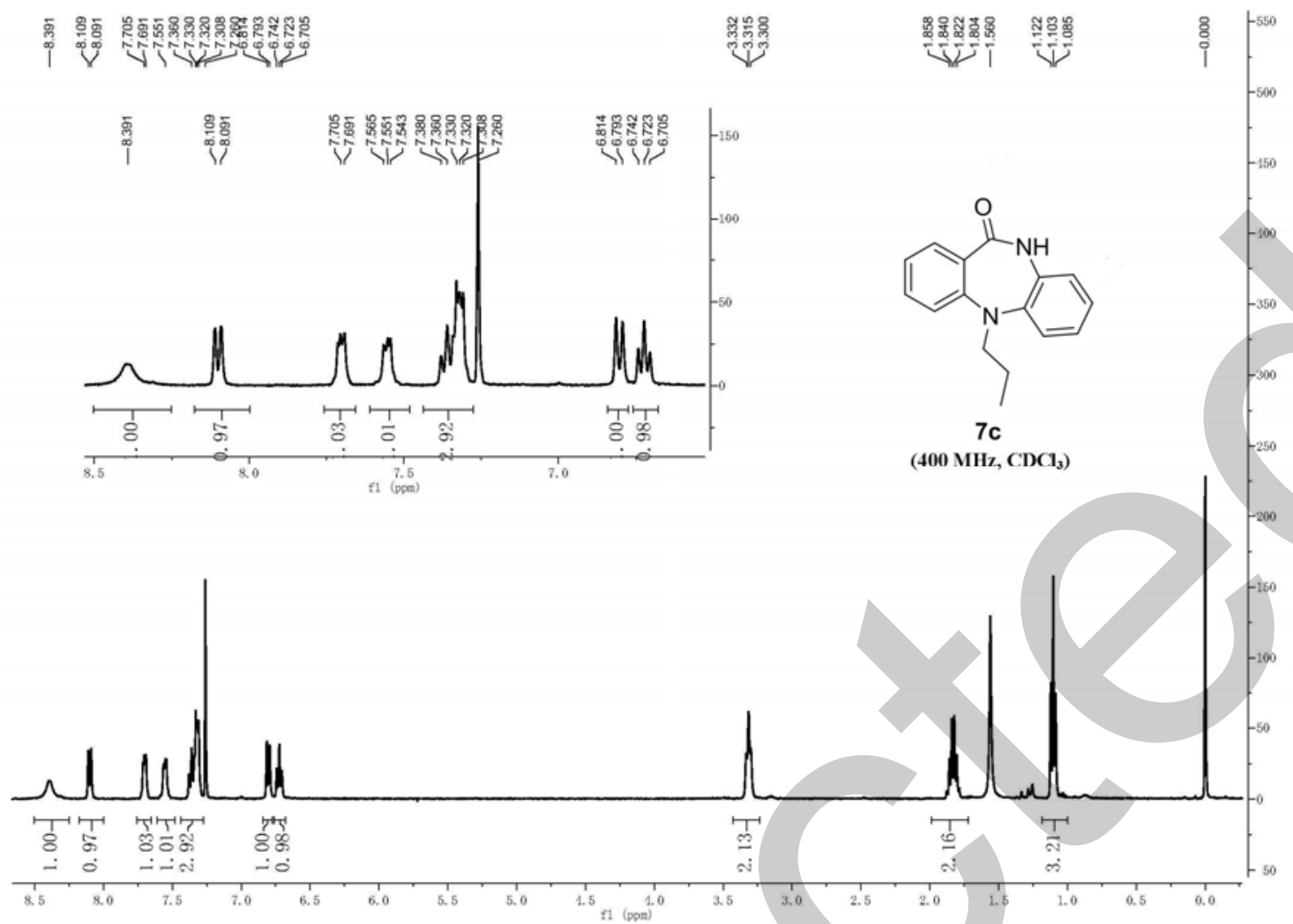

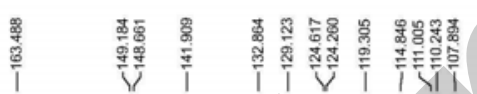

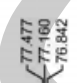

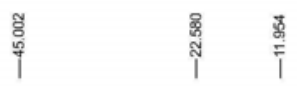
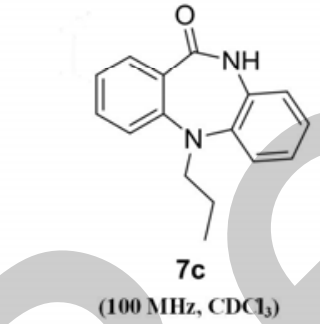

(100 $\left.\mathrm{MHz}_{2}, \mathrm{CDC1}_{3}\right)$
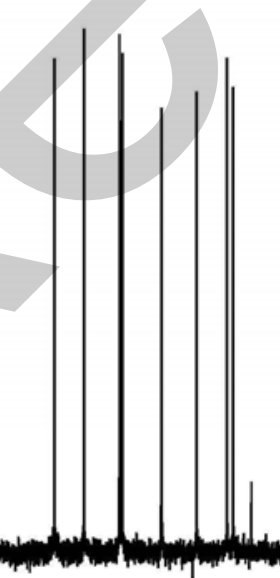

170

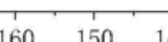

$10 \quad 130$

$120 \quad 110 \quad 100$

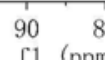

60

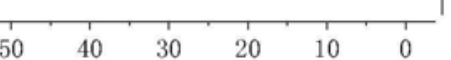




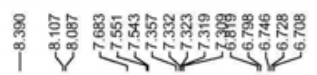

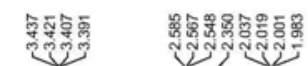

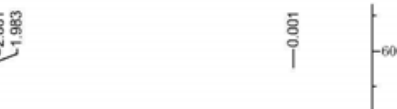

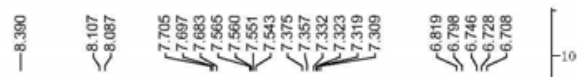
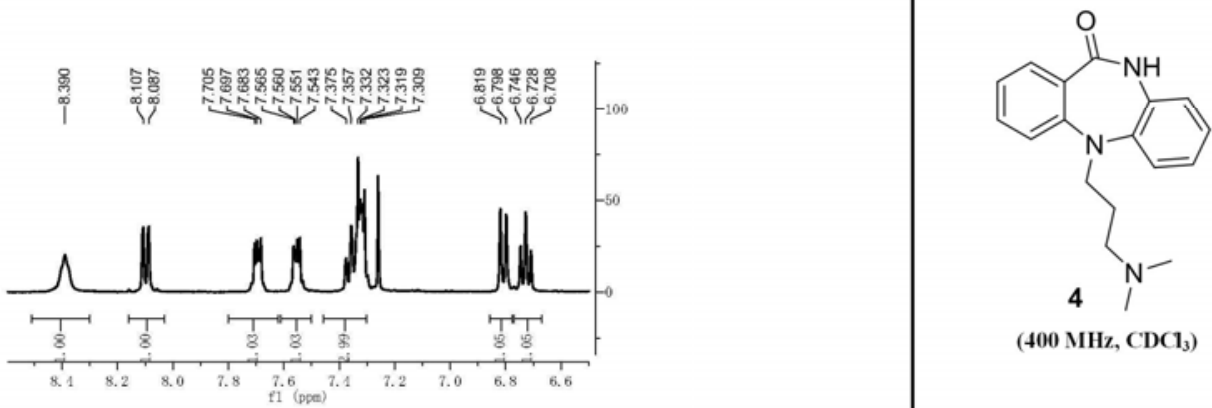

$\left(400 \mathrm{MHz}, \mathrm{CDC}_{3}\right)$

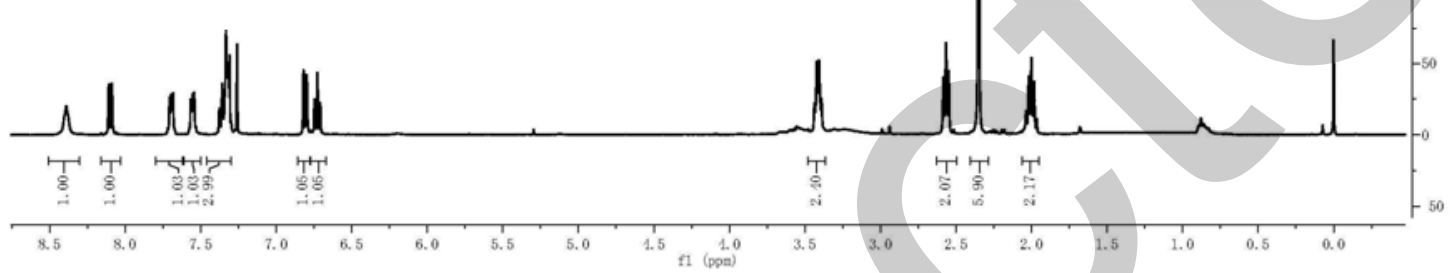

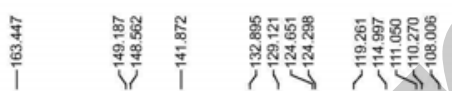
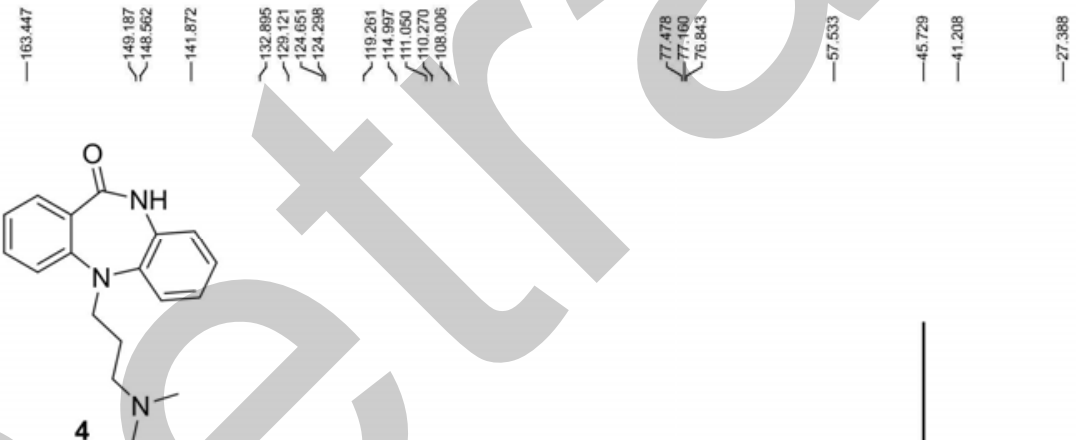

(100 $\left.\mathrm{MHz}, \mathrm{CDC1}_{3}\right)$
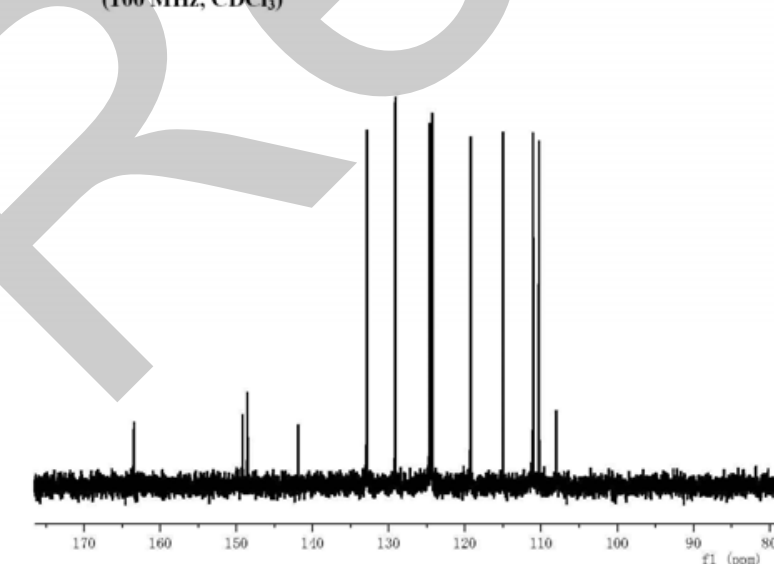

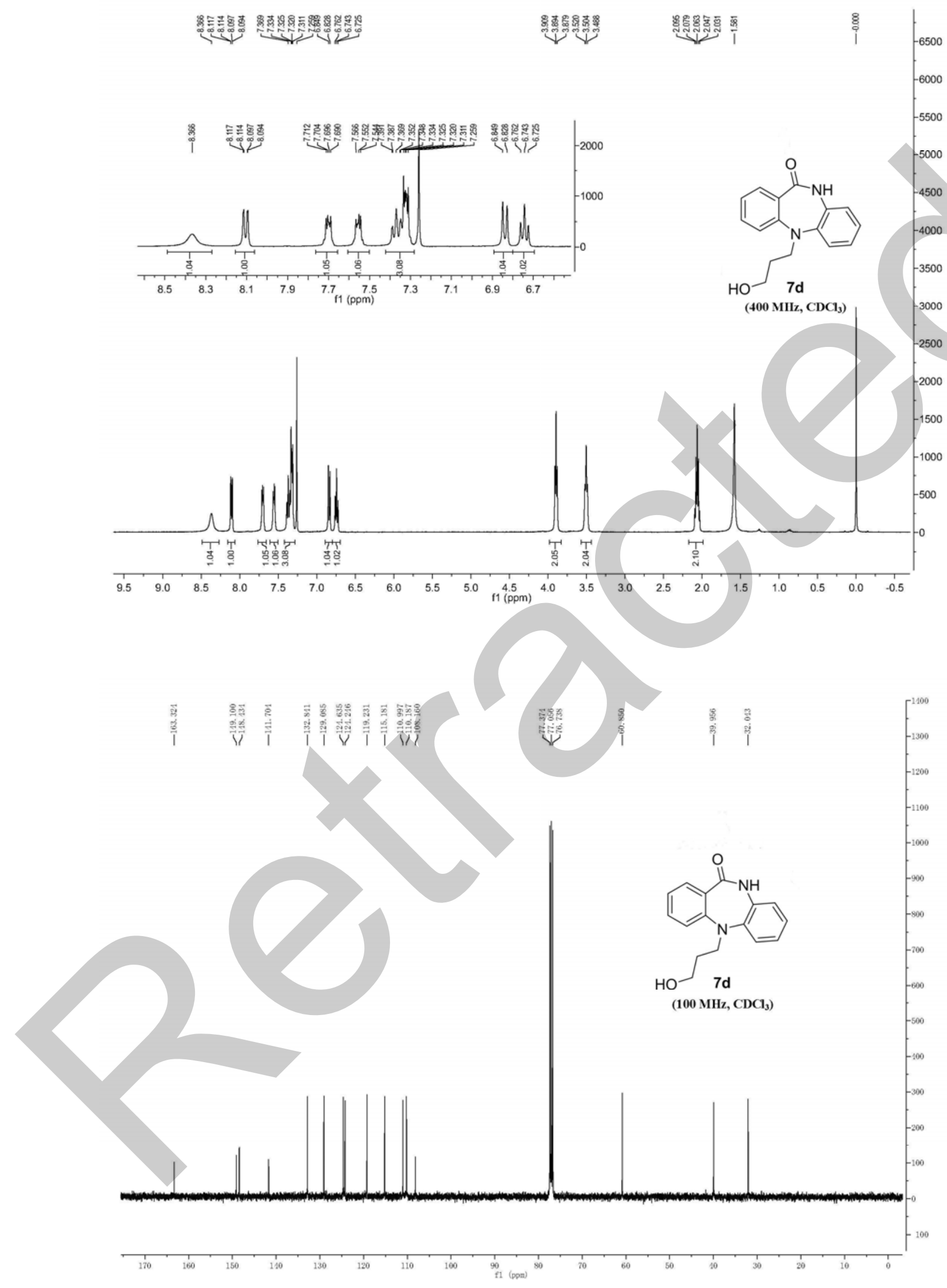


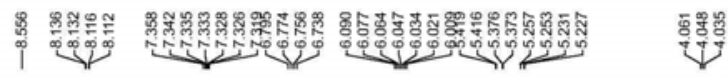
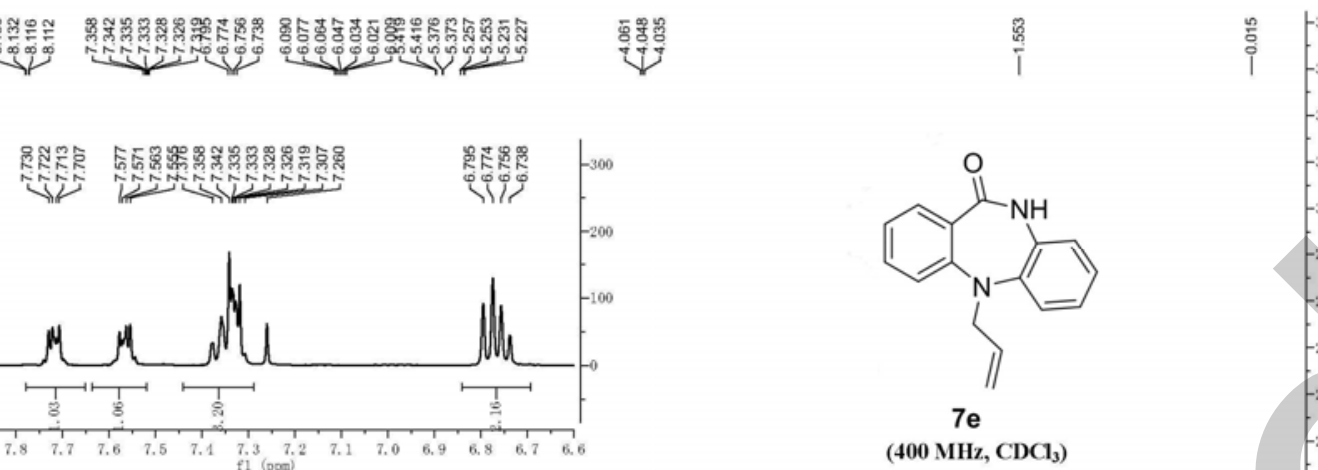

7 e

(400 $\left.\mathrm{MHz}, \mathrm{CDCl}_{3}\right)$

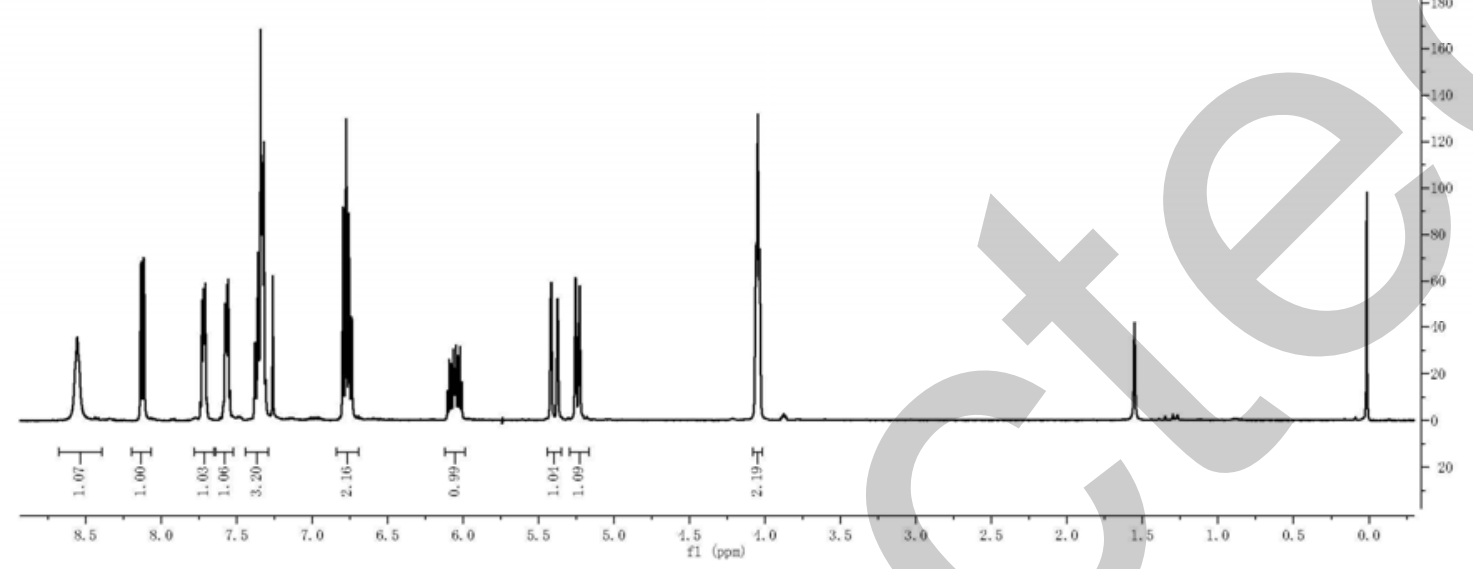

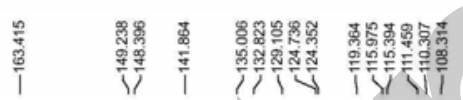

$\sqrt{8.98}$

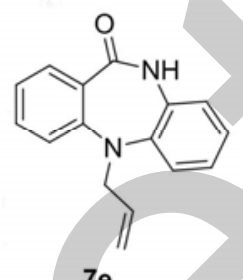

(100 MHz, $\left.\mathrm{CDCl}_{3}\right)$

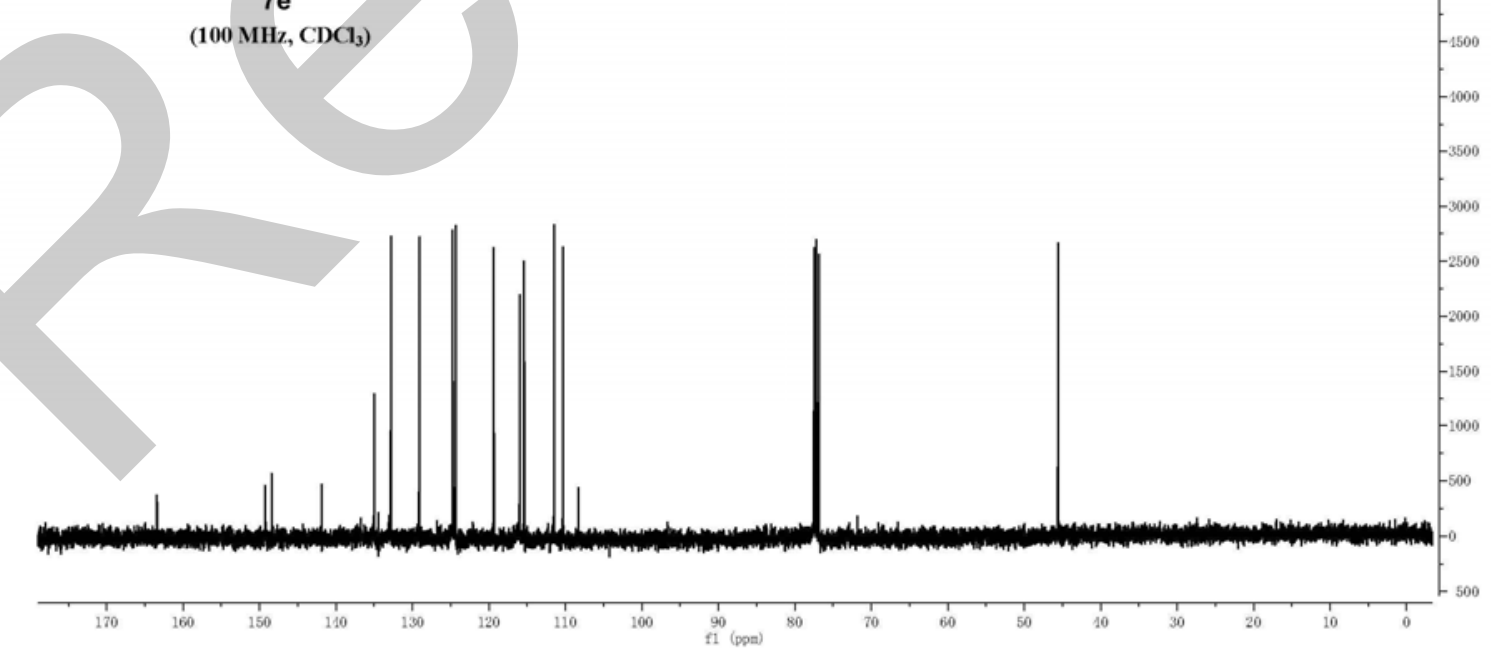




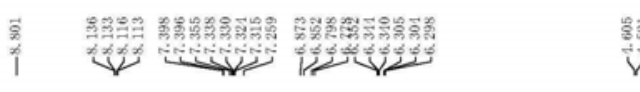

率
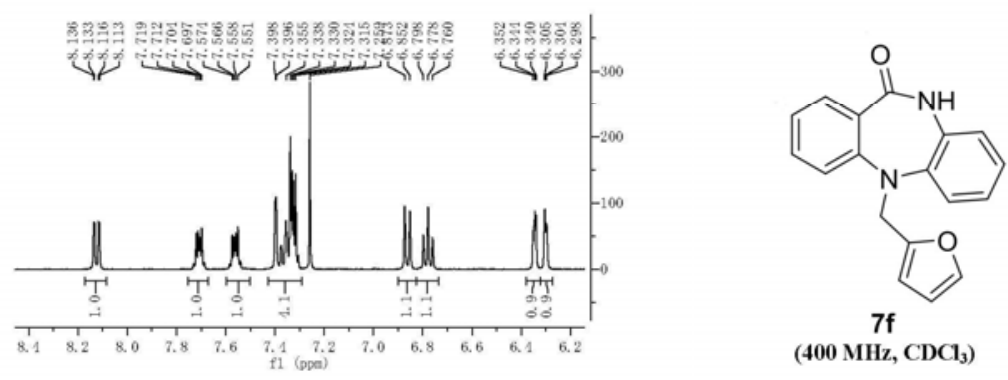

(400 $\left.\mathrm{MHz}, \mathrm{CDCl}_{3}\right)$

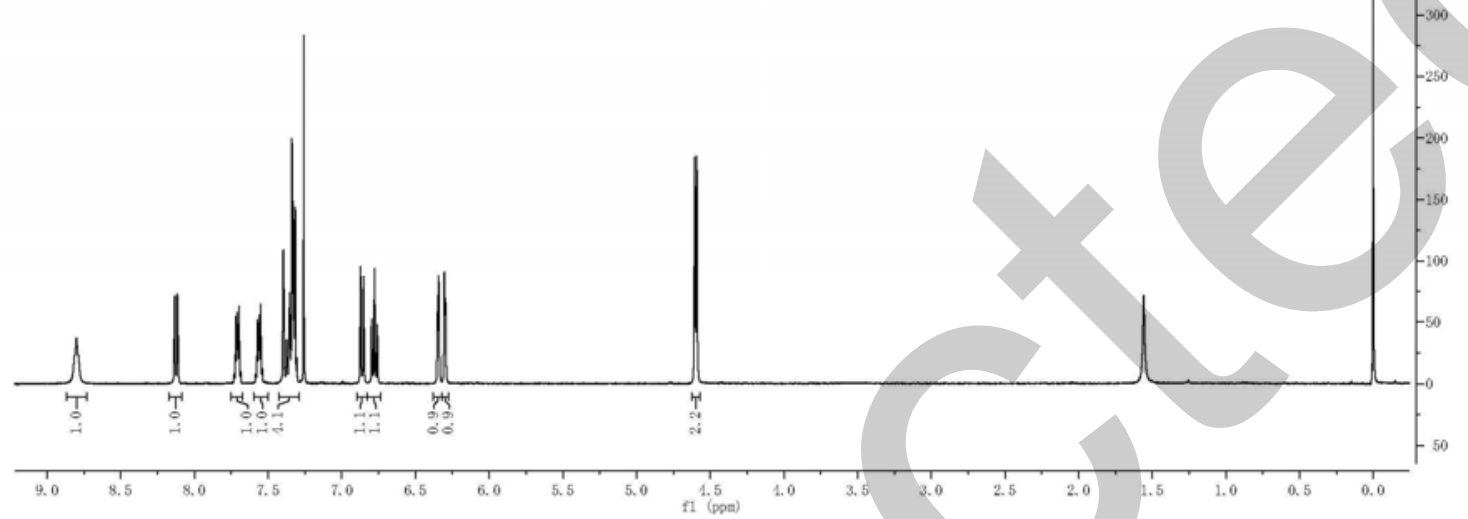

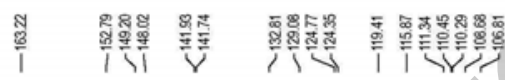

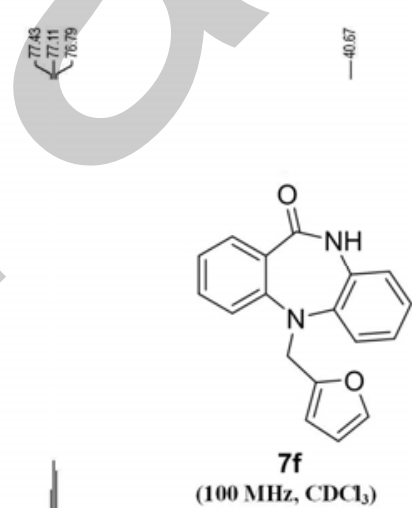

$7 f$

$\left(100 \mathrm{MHz}, \mathrm{CDCl}_{3}\right)$
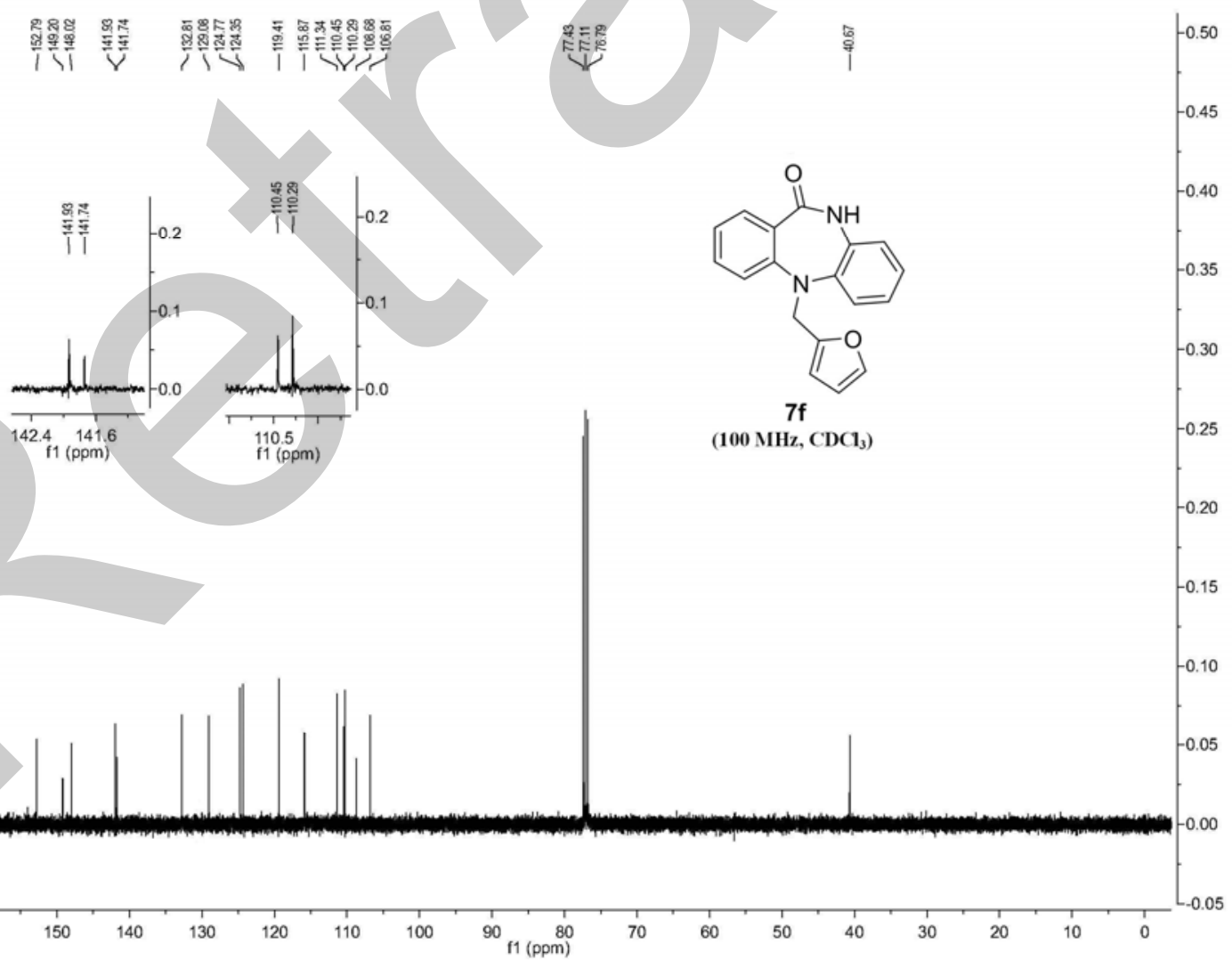


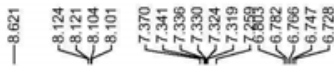
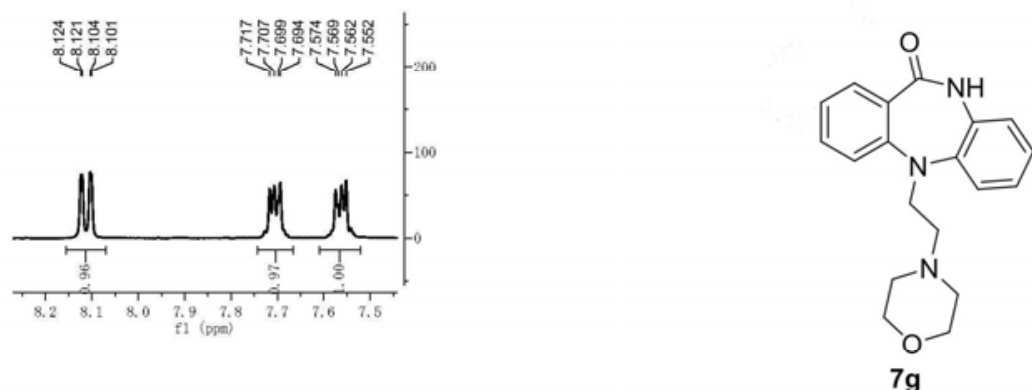

$\left(400 \mathrm{MHz}, \mathrm{CDC1}_{3}\right)$

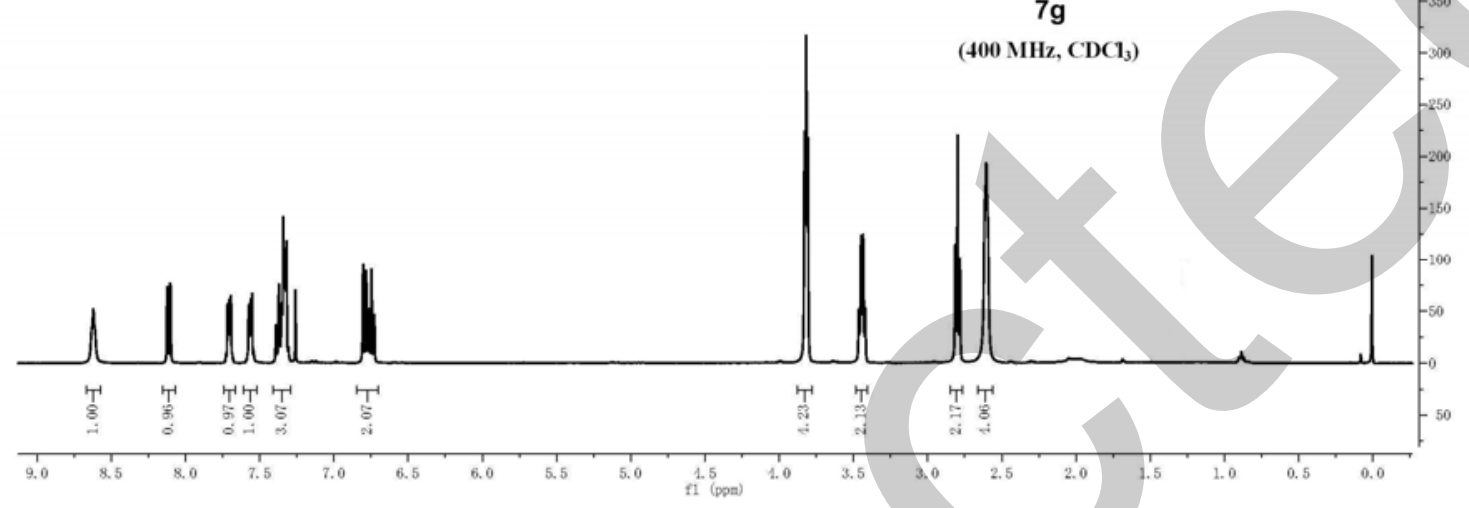

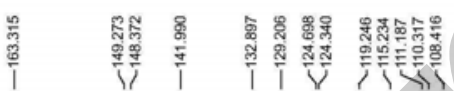

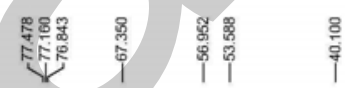
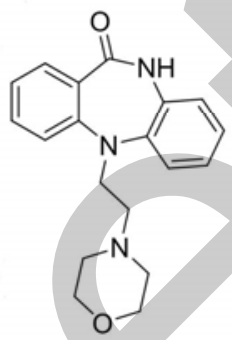

$7 \mathrm{~g}$

$\left(100 \mathrm{MHz}, \mathrm{CDCl}_{3}\right)$

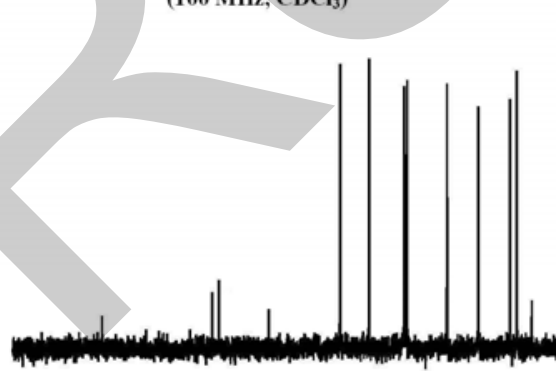

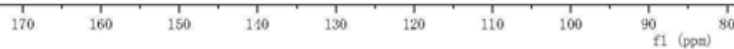




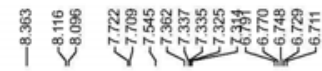

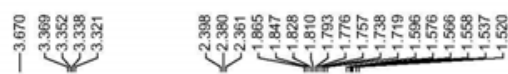
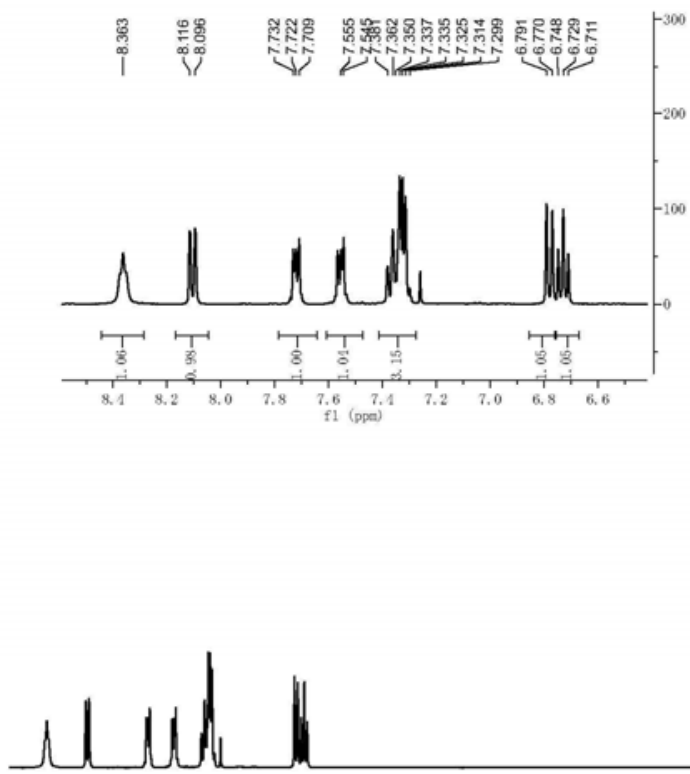

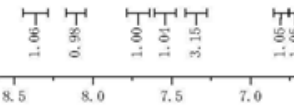

$\underbrace{+1}_{6.5}$

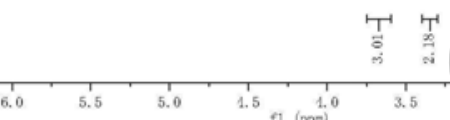

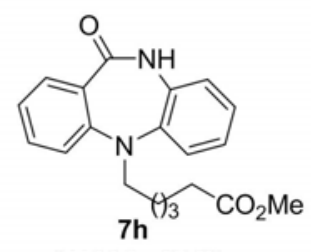

(400 MHz, $\mathrm{CDCl}_{3}$ )

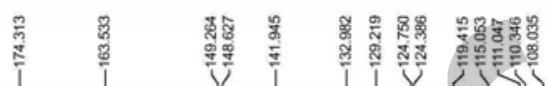

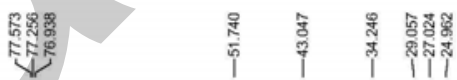

\begin{tabular}{c}
-3200 \\
-3000 \\
\hline-2800 \\
\hline-2600 \\
\hline-2100 \\
\hline-2200 \\
\hline-2000 \\
\hline-1800 \\
\hline-1600 \\
\hline-1100 \\
\hline-1200 \\
\hline-1000 \\
\hline-800 \\
\hline-200 \\
\hline-100 \\
\hline-200 \\
\hline-
\end{tabular}
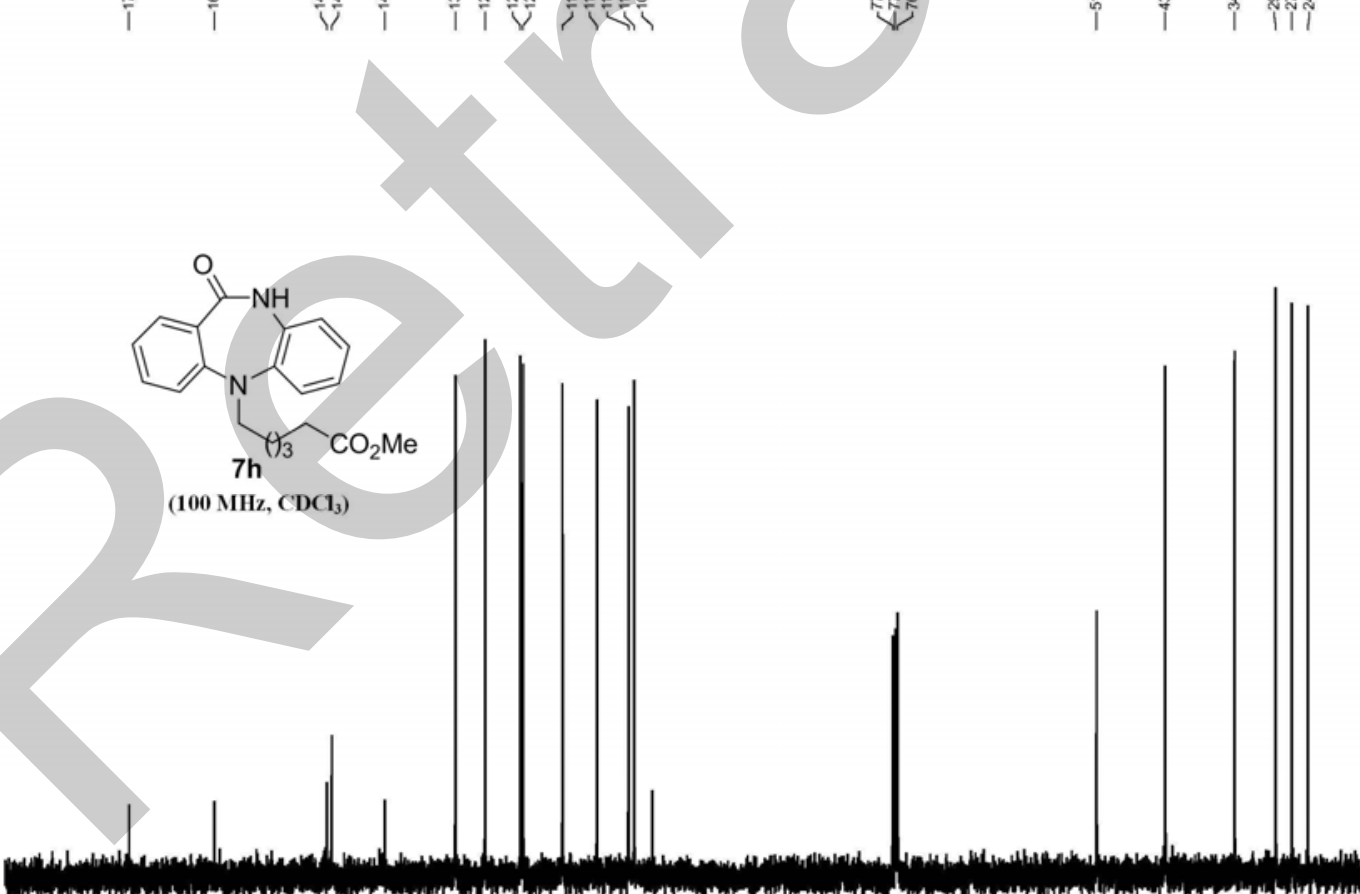


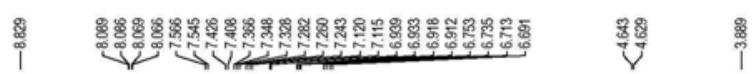
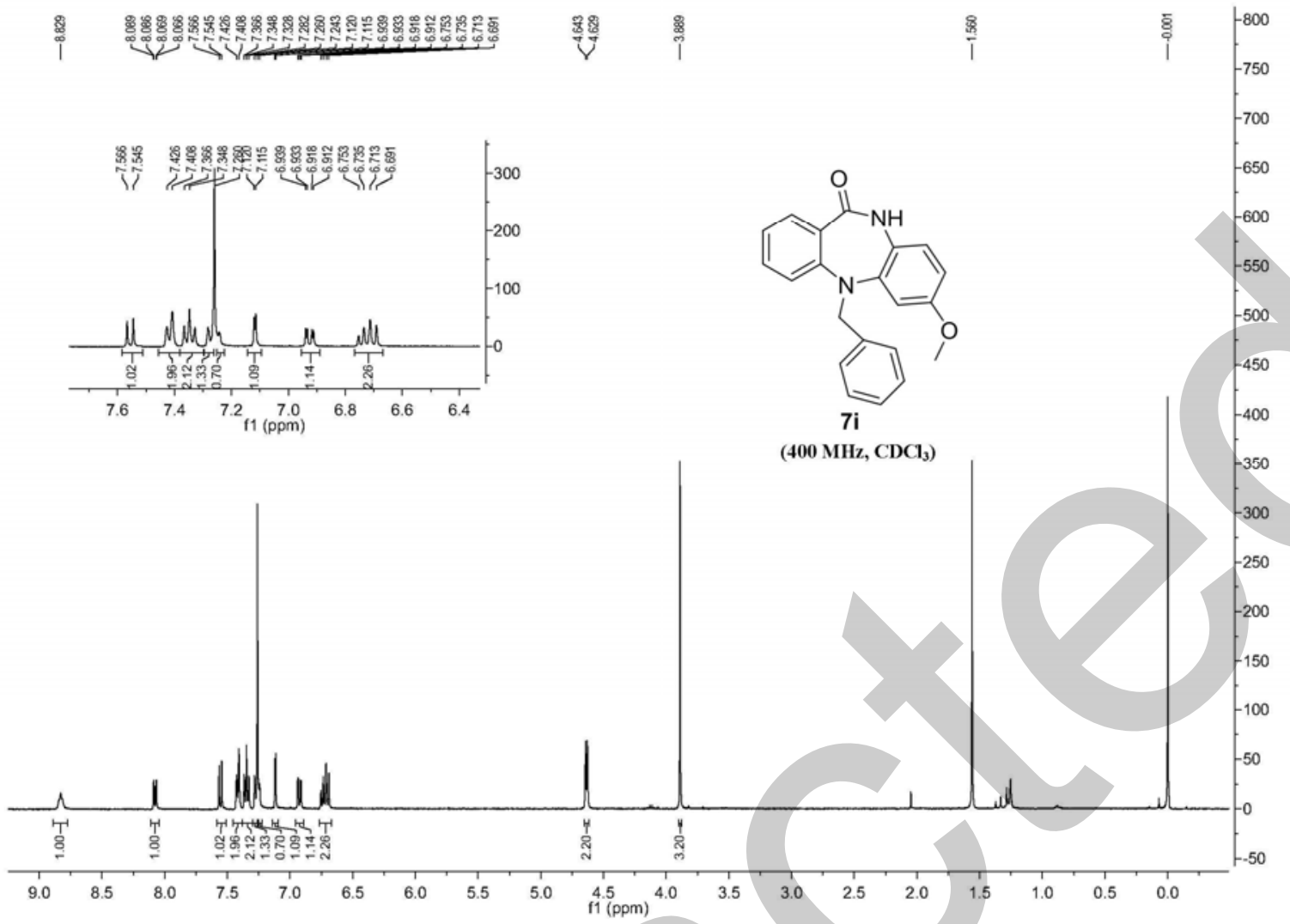

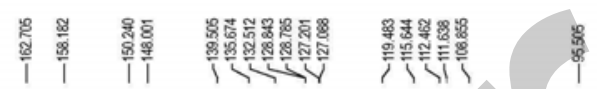
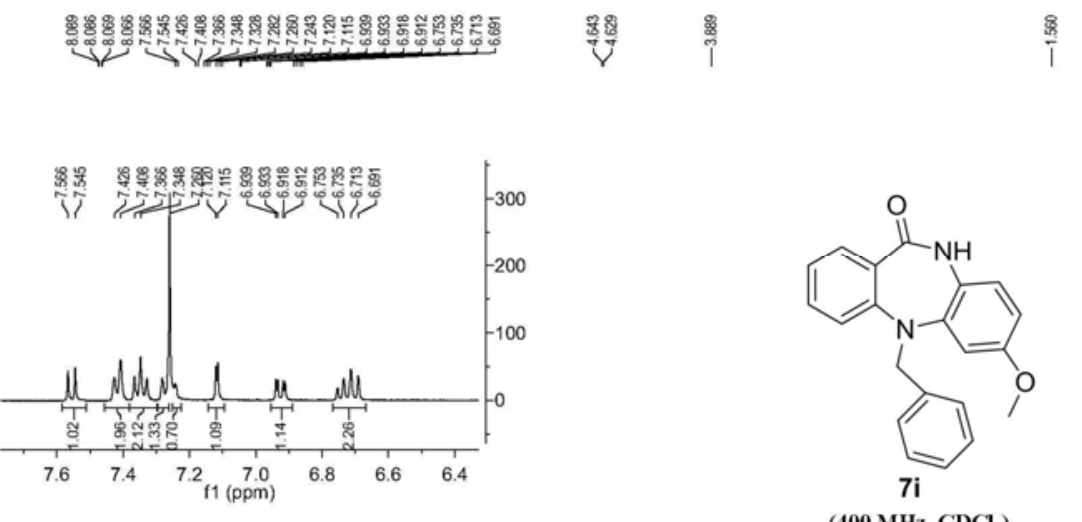


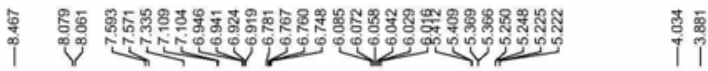
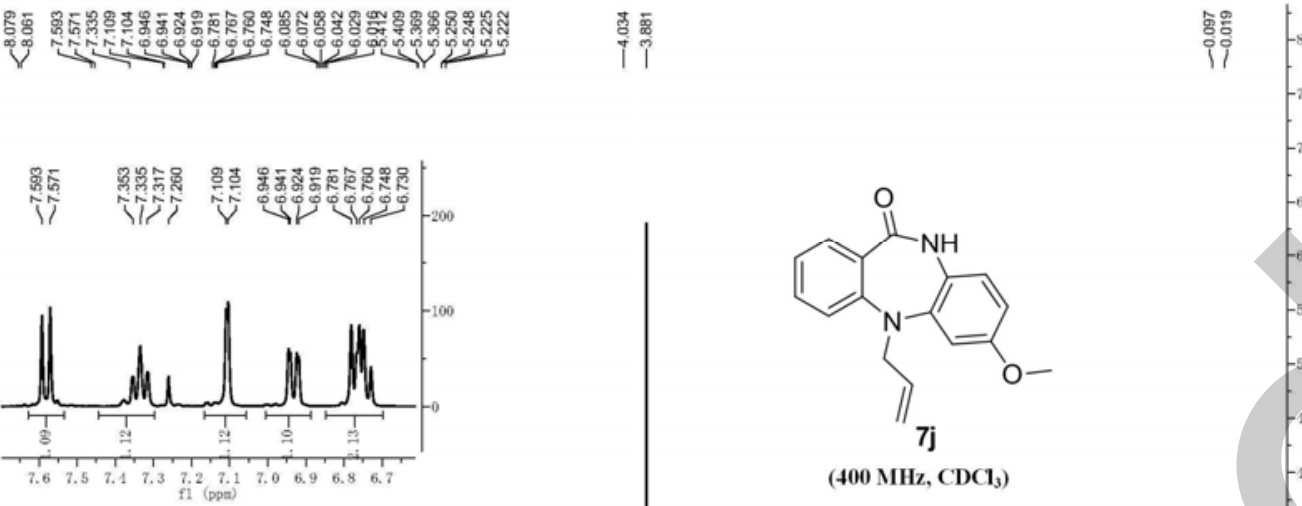

$\left(400 \mathrm{MHz}_{2} \mathrm{CDC1}_{3}\right)$

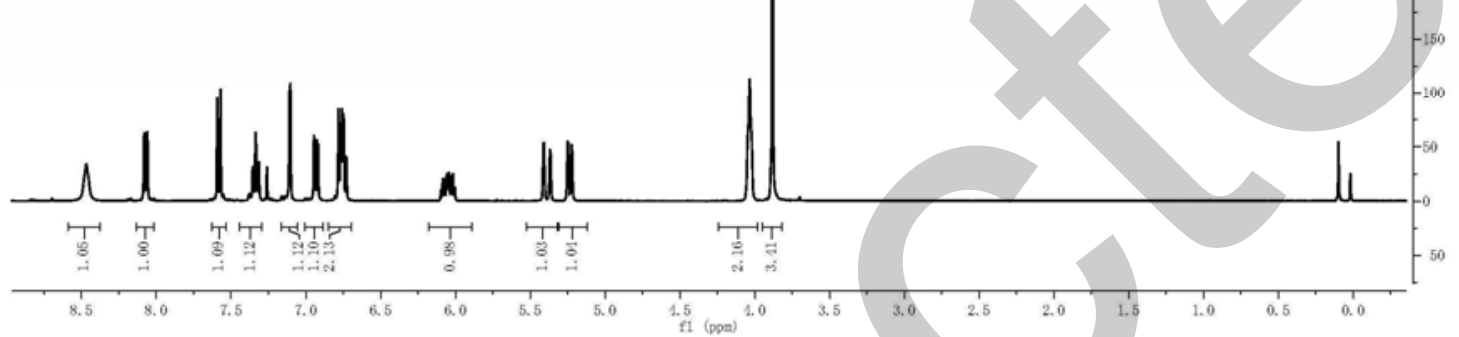

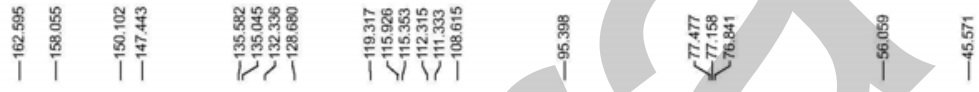
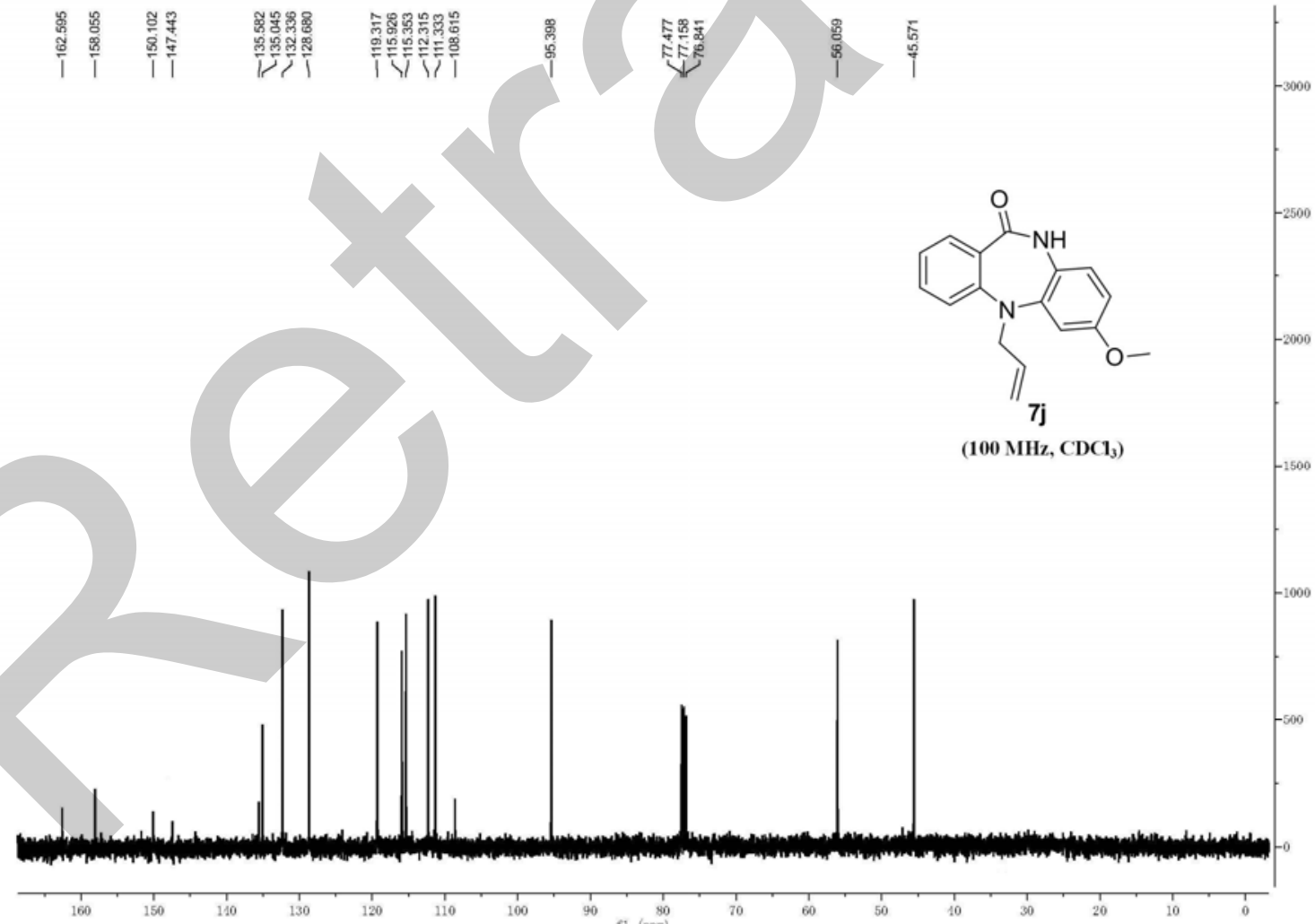


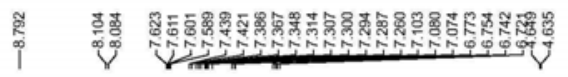
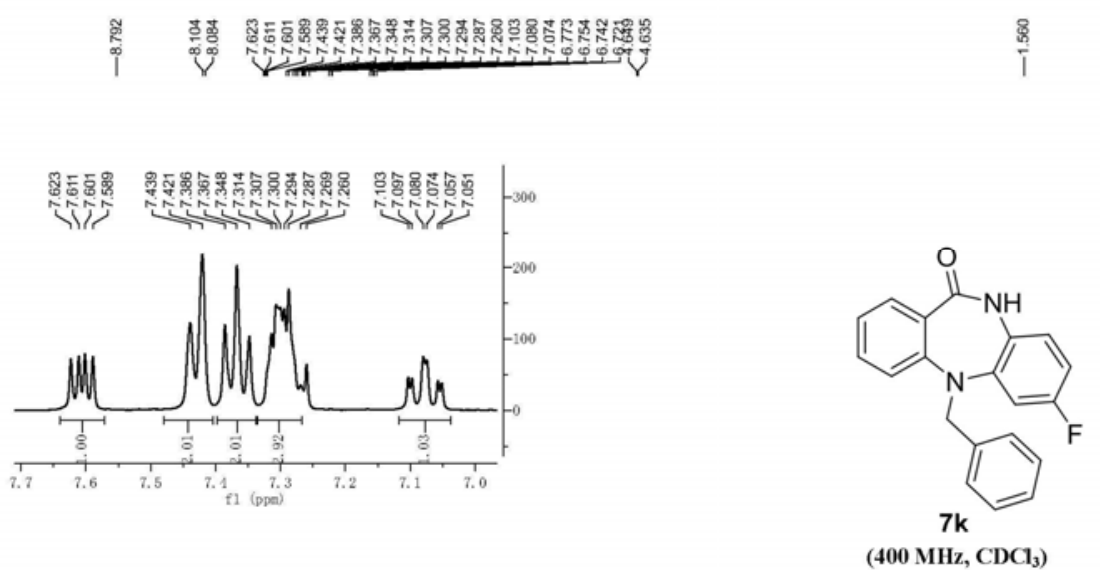

$\left(400 \mathrm{MHz}, \mathrm{CDCl}_{3}\right)$

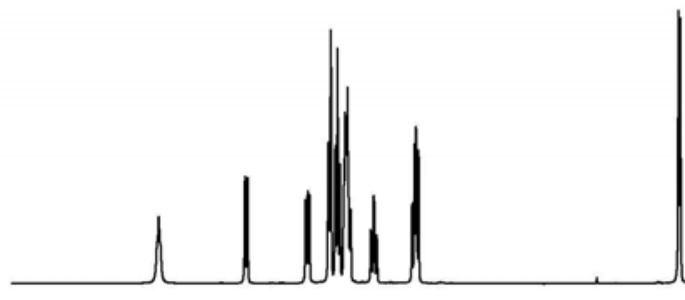

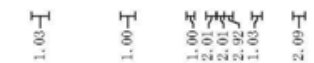

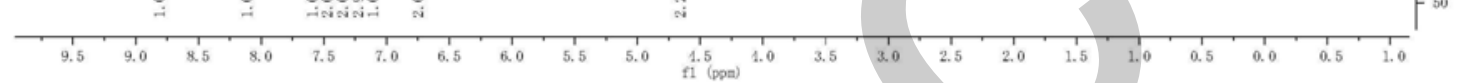

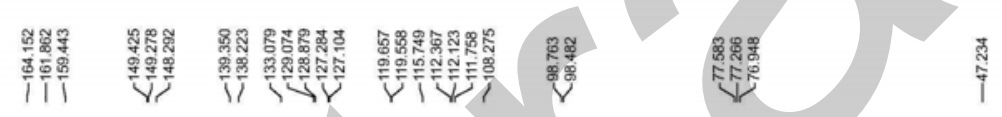
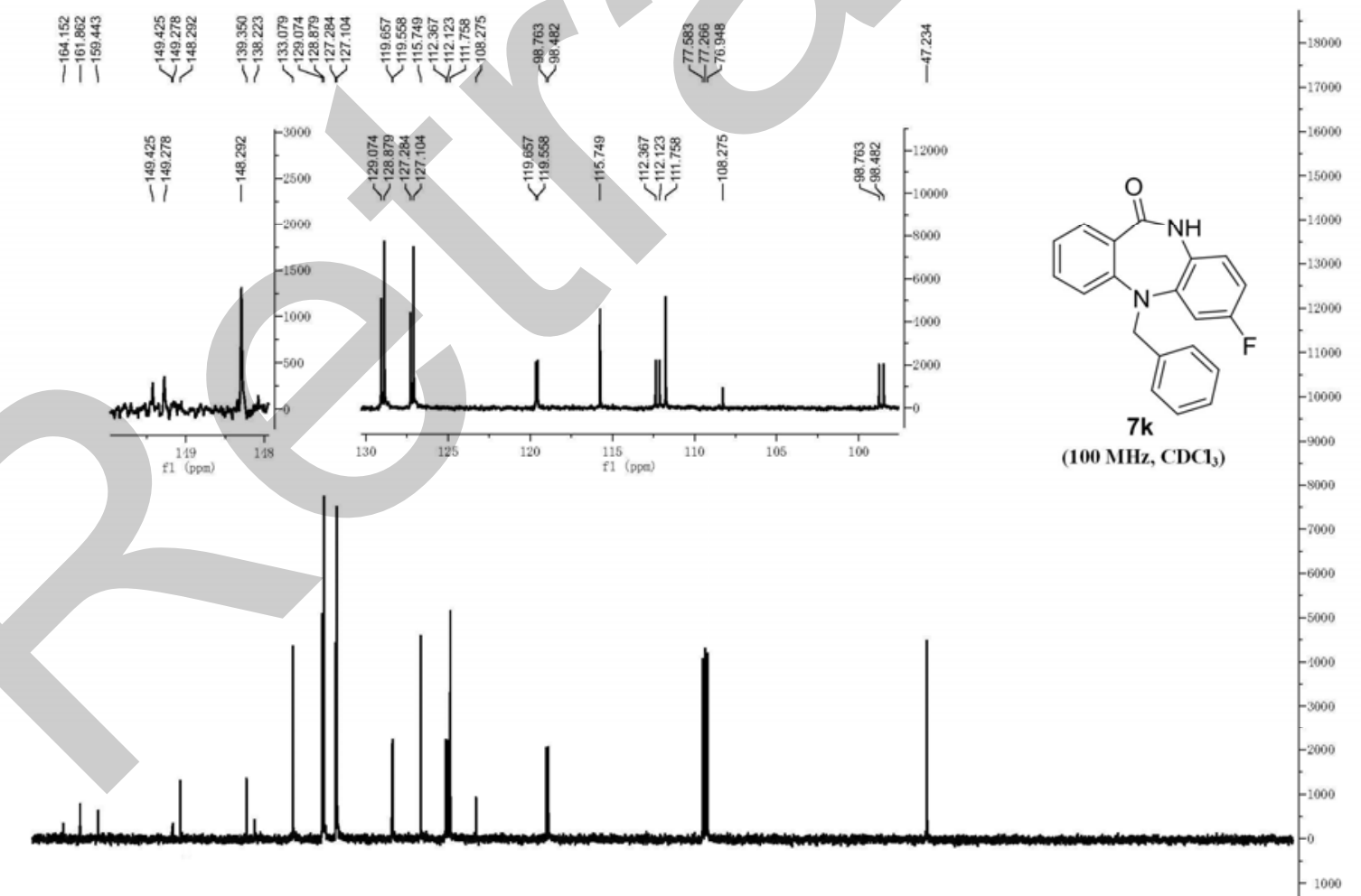

$\left.\mathrm{MHz}, \mathrm{CDCl}_{3}\right)$ 


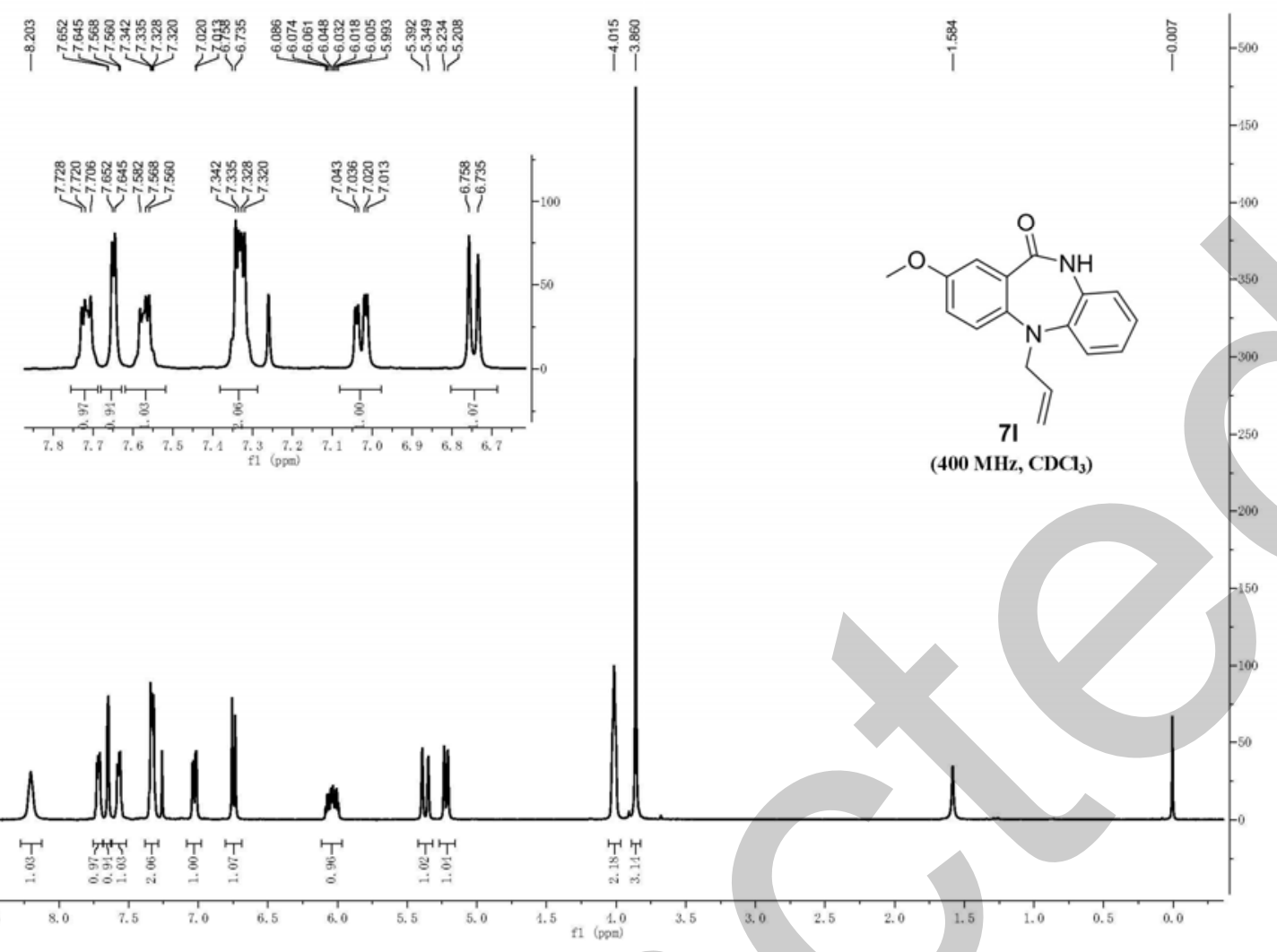

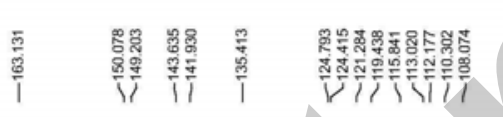
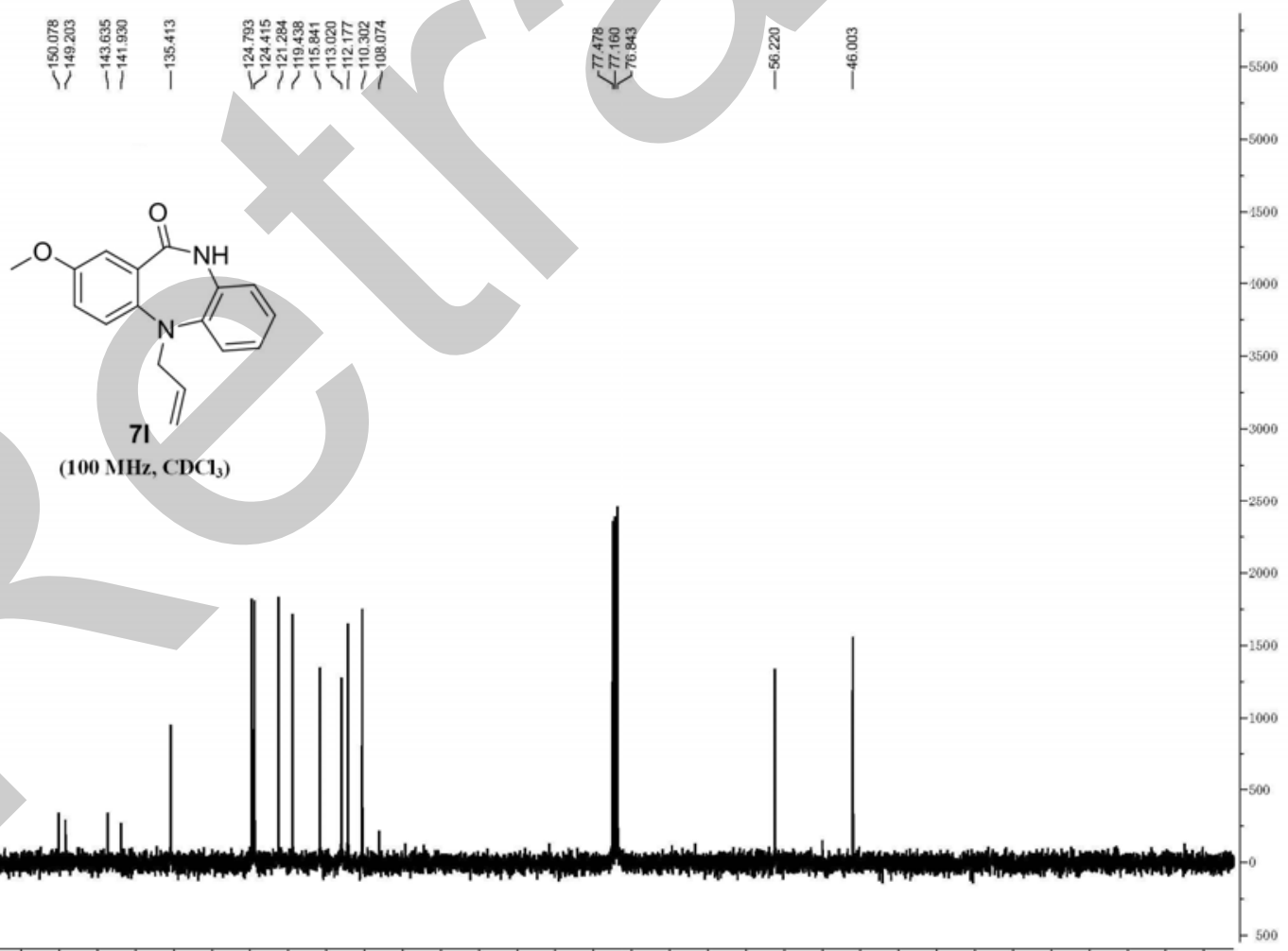


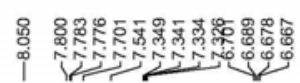

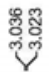

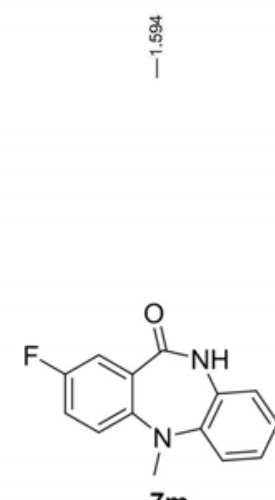

휴ํำ ${ }^{-1400}$

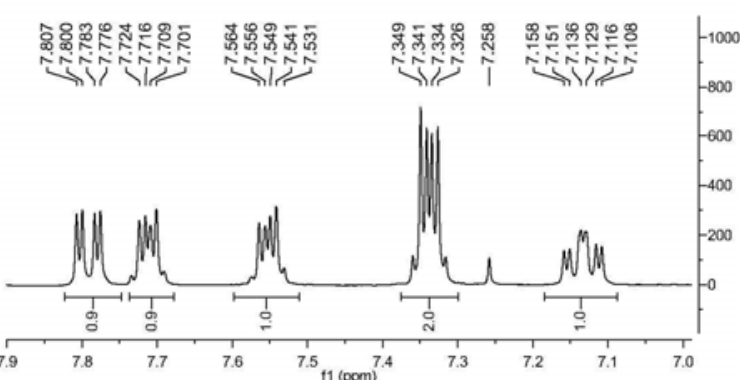

(400 MHz, $\mathrm{CDCl}_{3}$ )

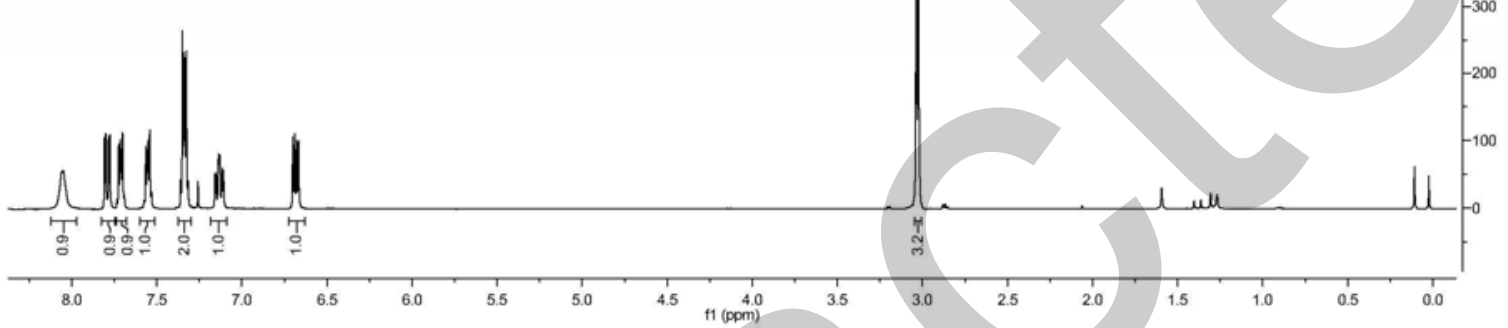

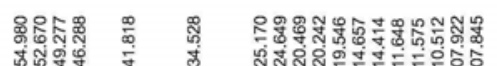

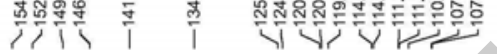
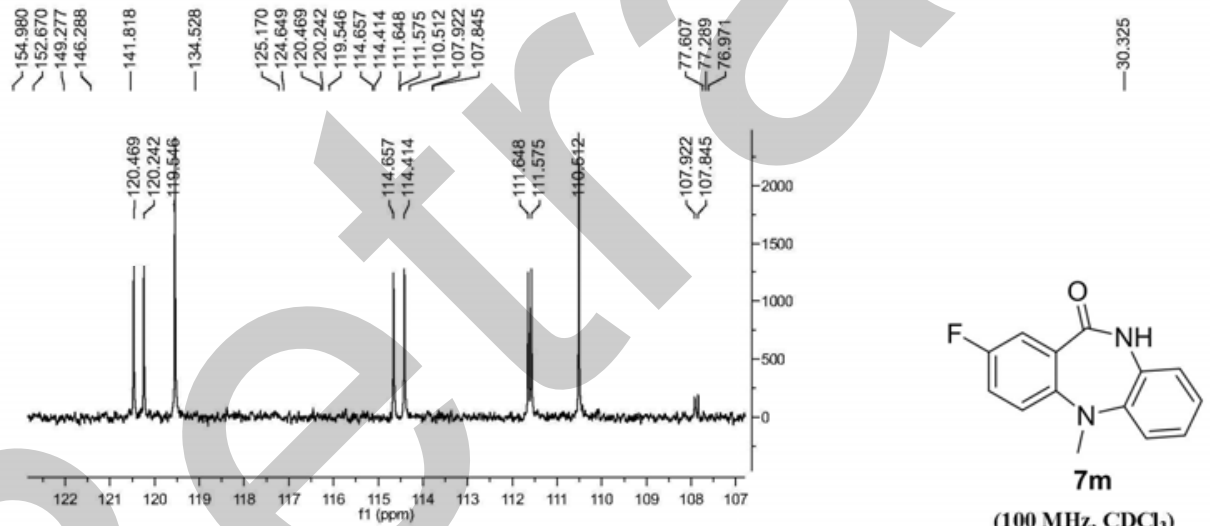

$-0500$

(100 $\mathrm{MHz}, \mathrm{CDCl}_{3}$ )

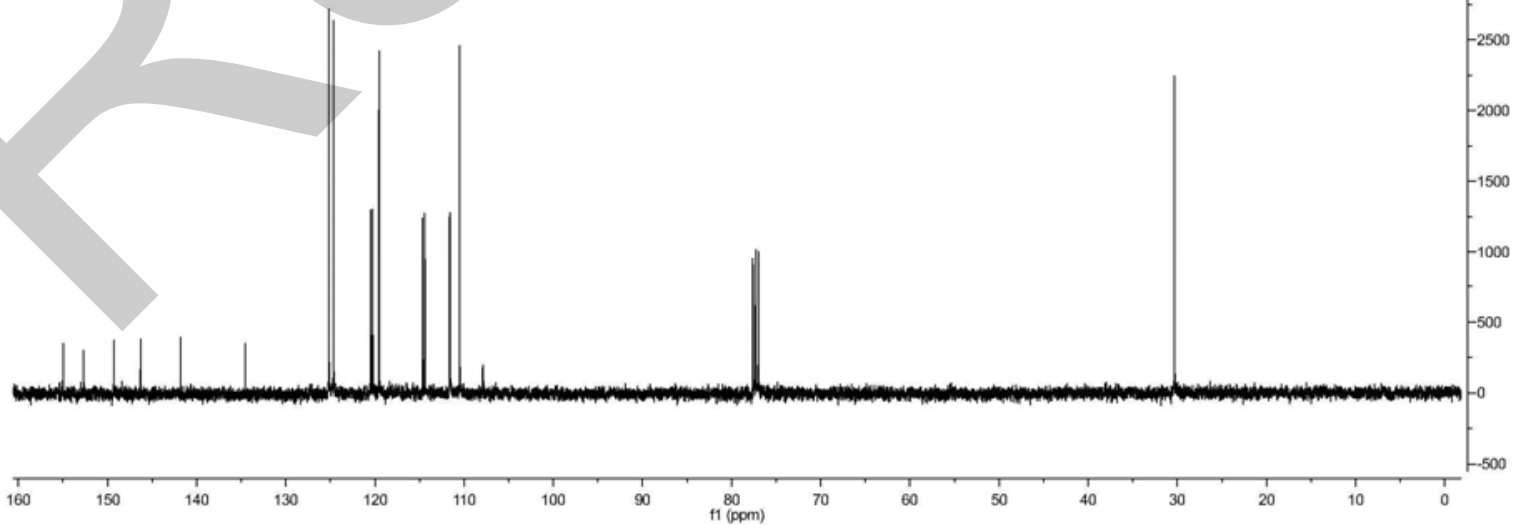


兽

ฟू๊

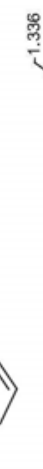

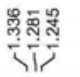

: i $^{-2400}$

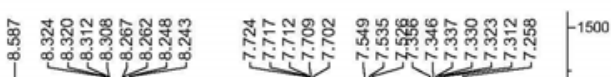<smiles>CN1c2ccccc2NC(=O)c2cccnc21</smiles>

$7 n$

(400 MHz, $\left.\mathrm{CDCl}_{3}\right)$
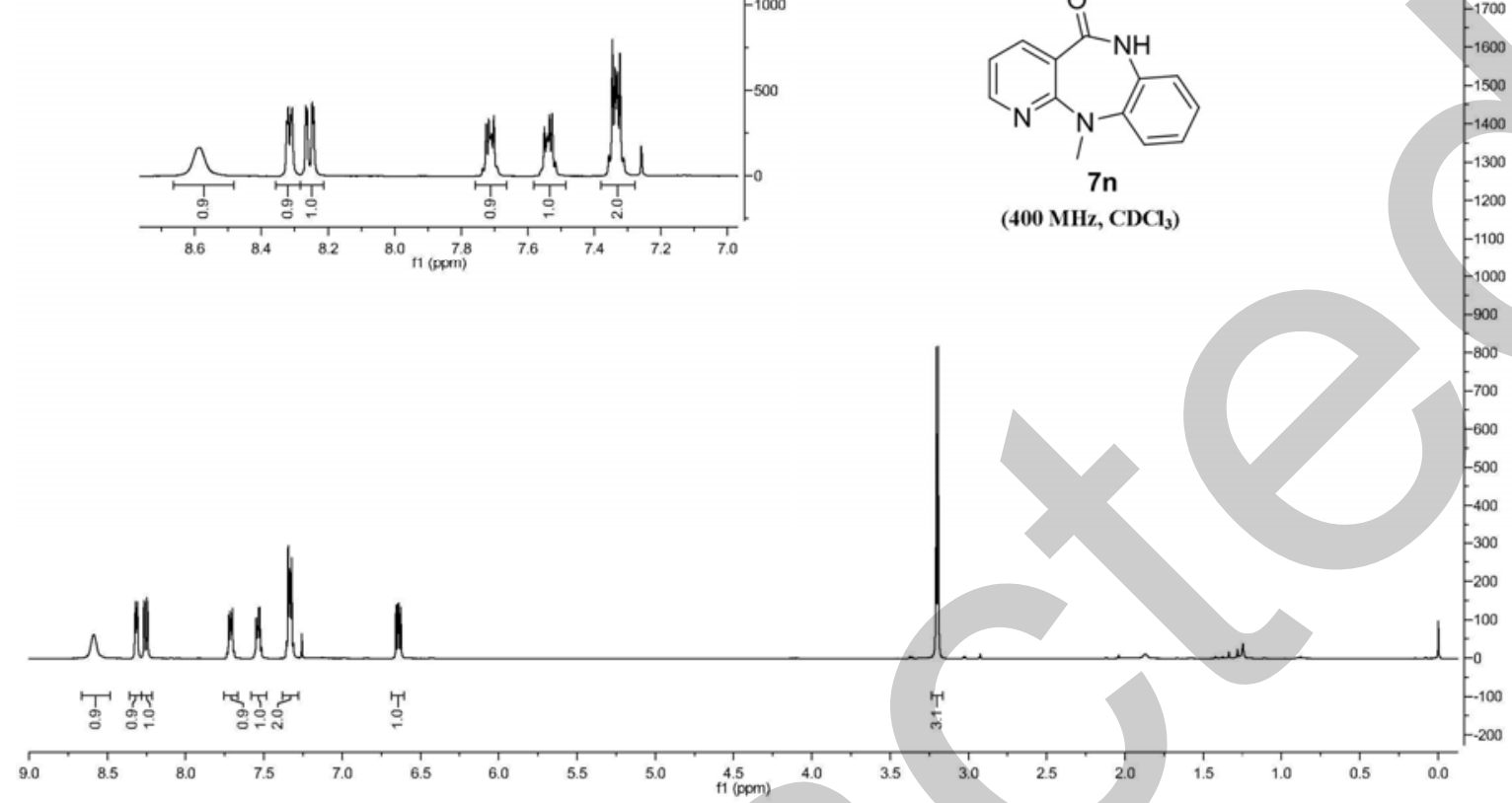

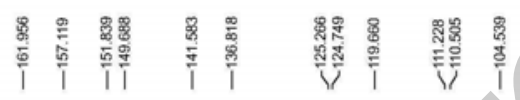
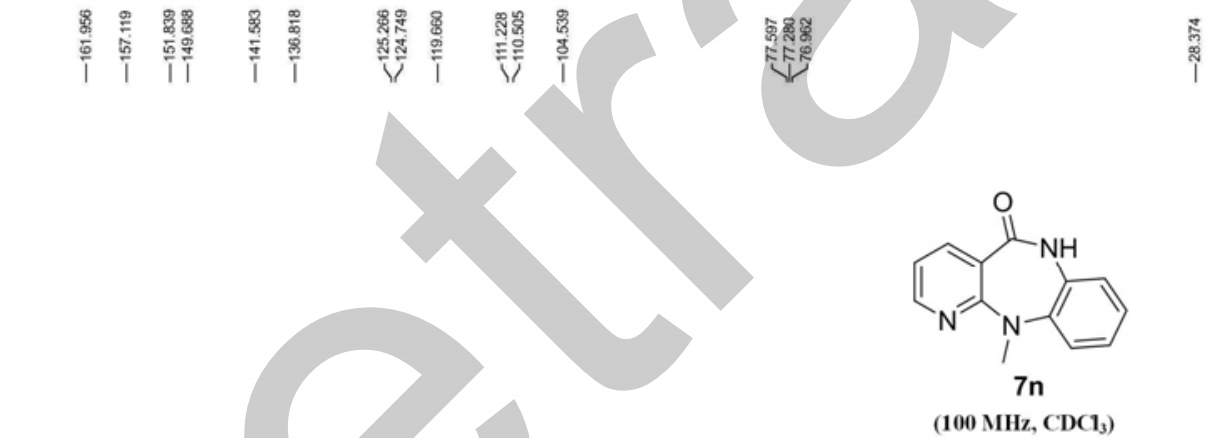

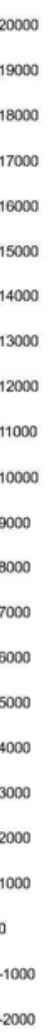
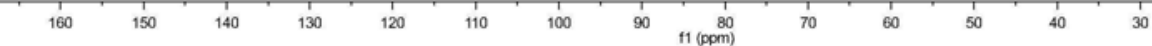


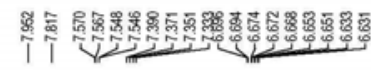

繁

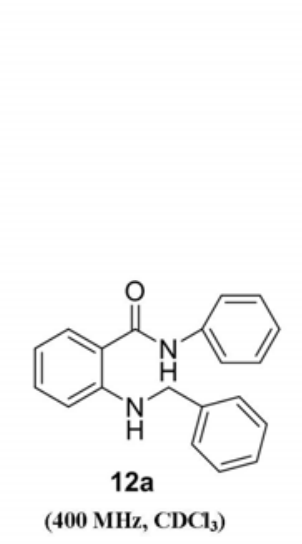

$\left(400 \mathrm{MHz}, \mathrm{CDCl}_{3}\right)$
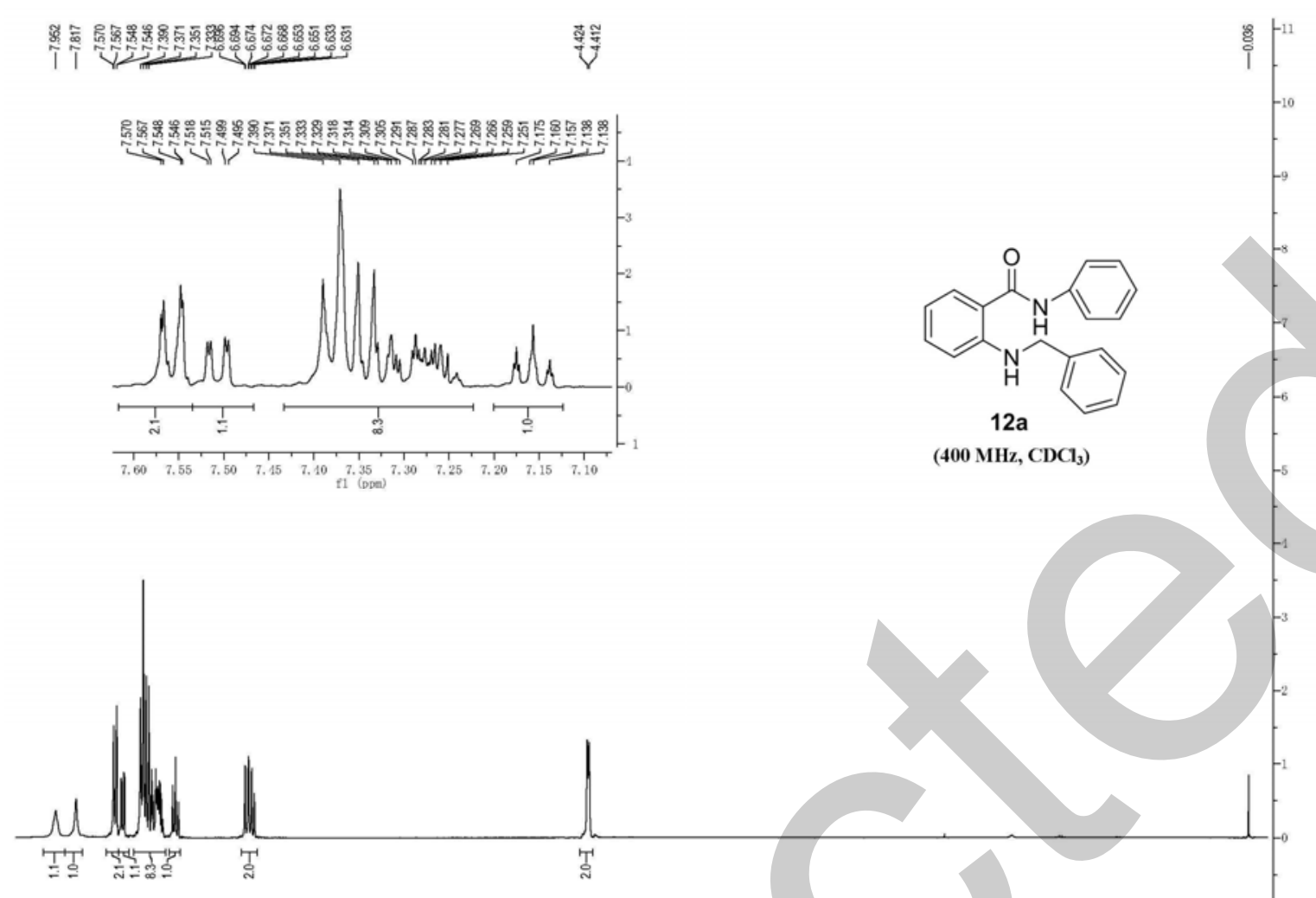

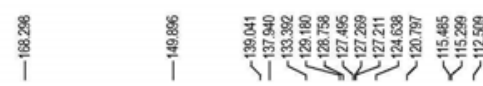

器㩊

买
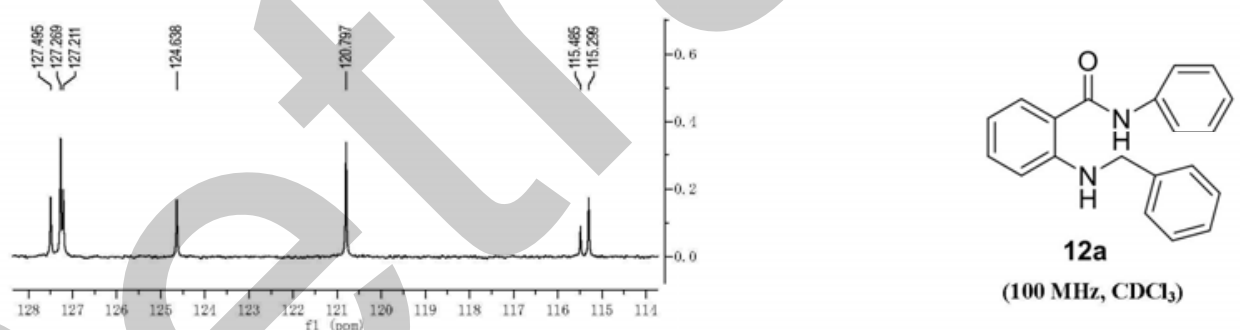

12a

(100 MHz, $\mathrm{CDCl}_{3}$ )

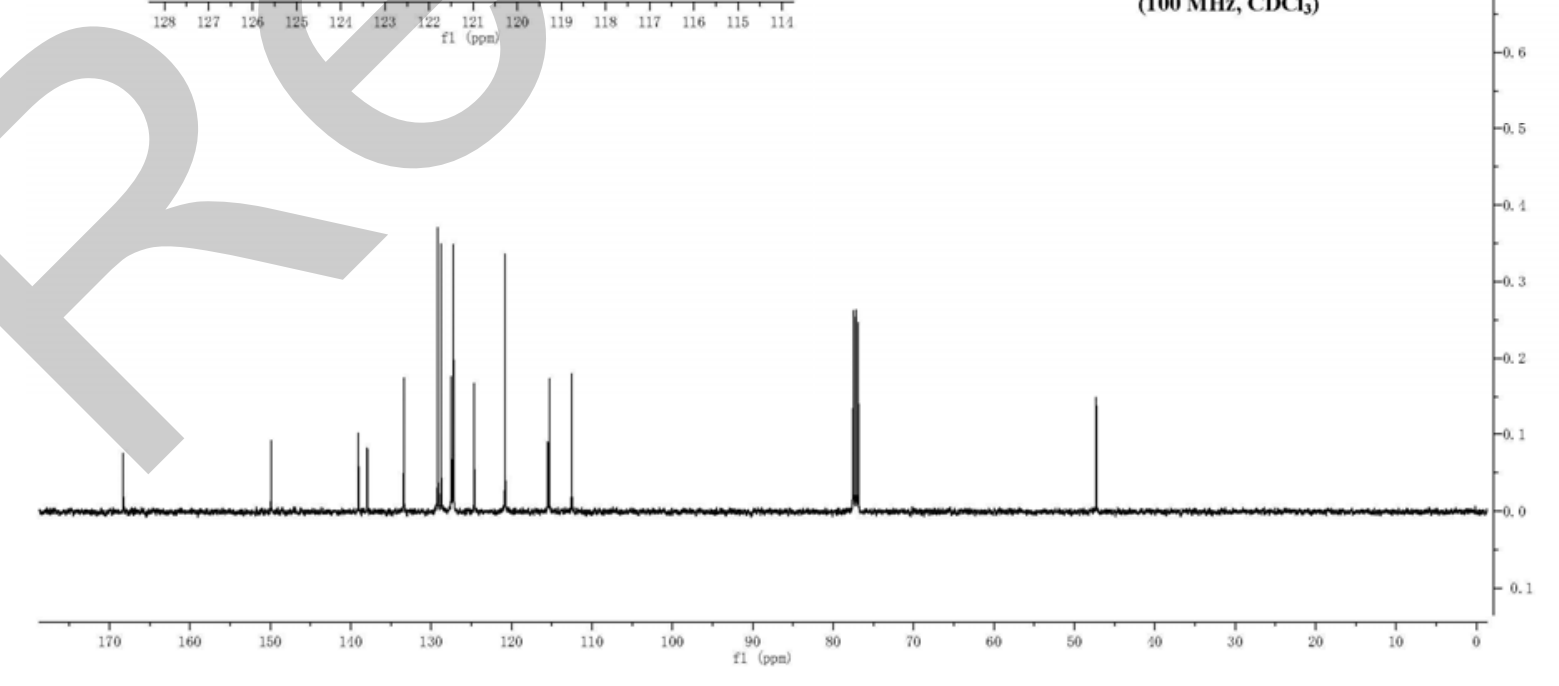




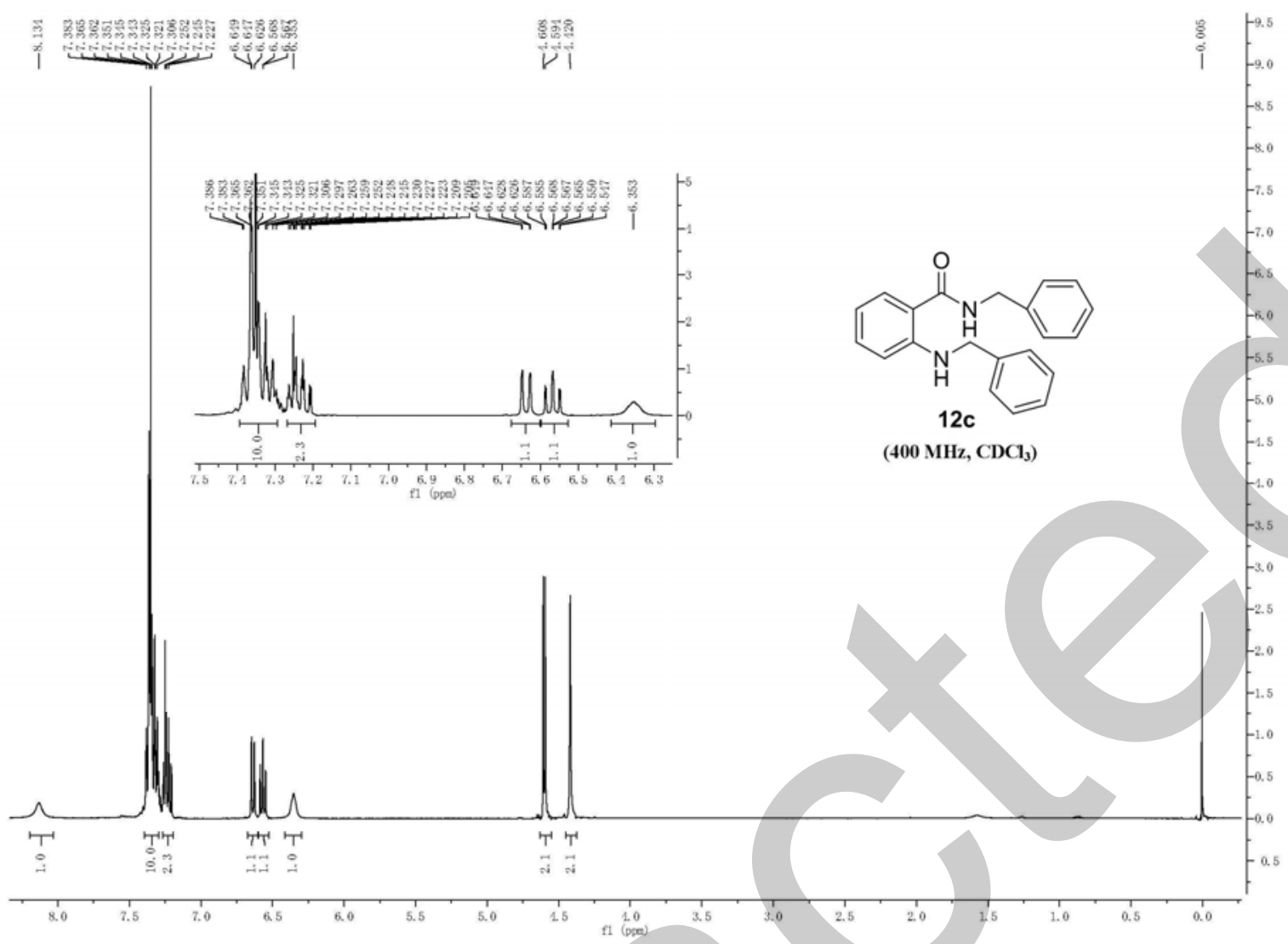

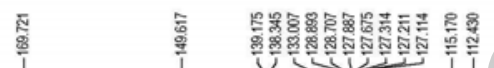

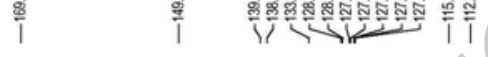

哭哭
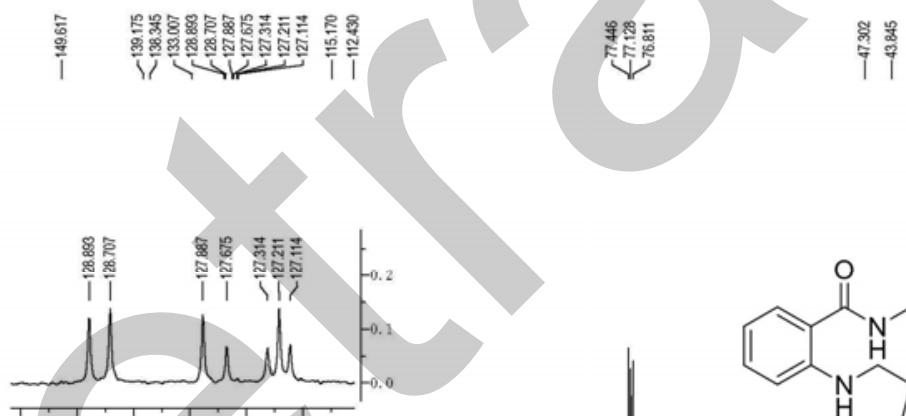

129.5 $129.0128 .5 \quad 128.0 \quad 127.5$ 127.0
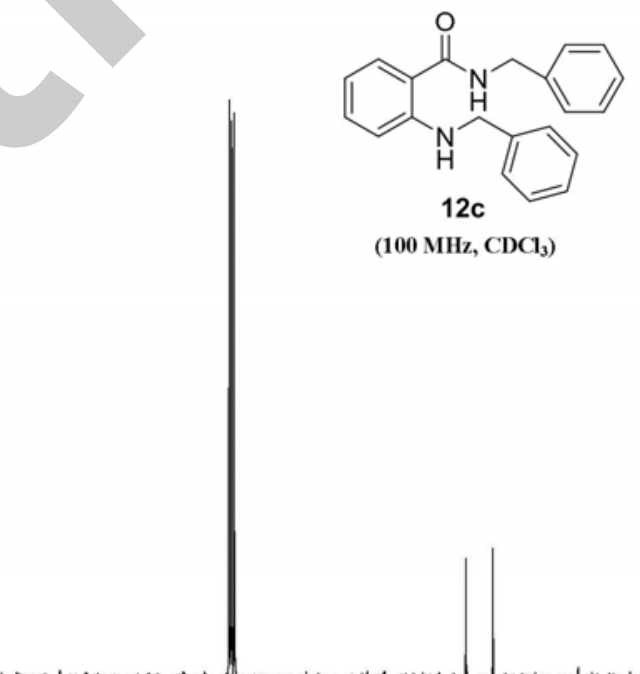

器善

(100 MHz, $\left.\mathrm{CDCl}_{3}\right)$

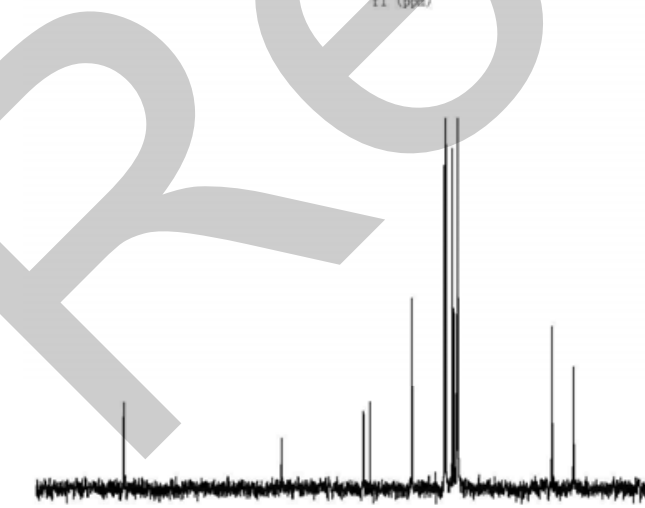


<smiles>O=C(Nc1ccc(Cl)cc1)c1ccccc1NCc1ccccc1</smiles>

12d

(400 MHz, $\left.\mathrm{CDC1}_{3}\right)$

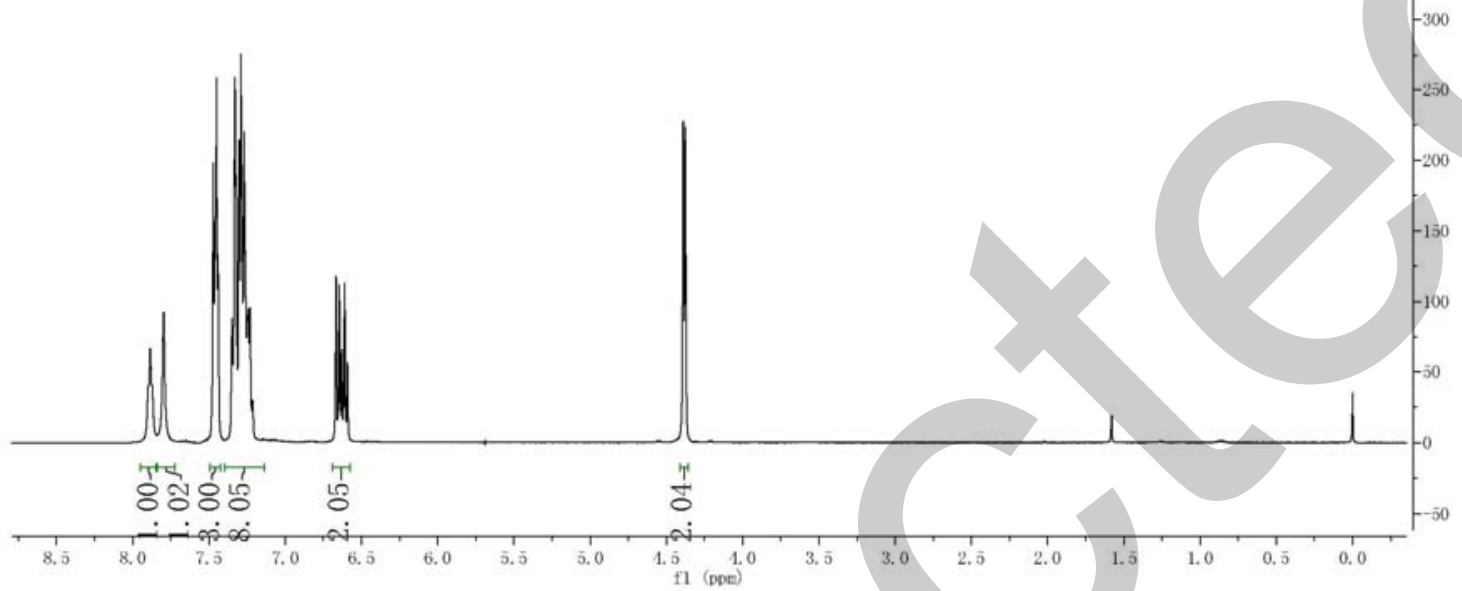

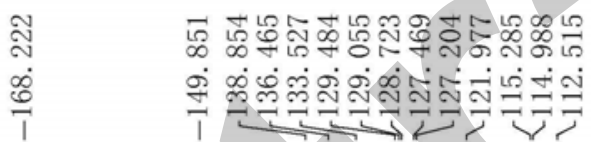

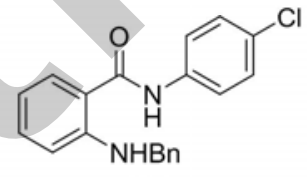

$12 d$

$\left(100 \mathrm{MHz}, \mathrm{CDCl}_{\mathrm{j}}\right.$
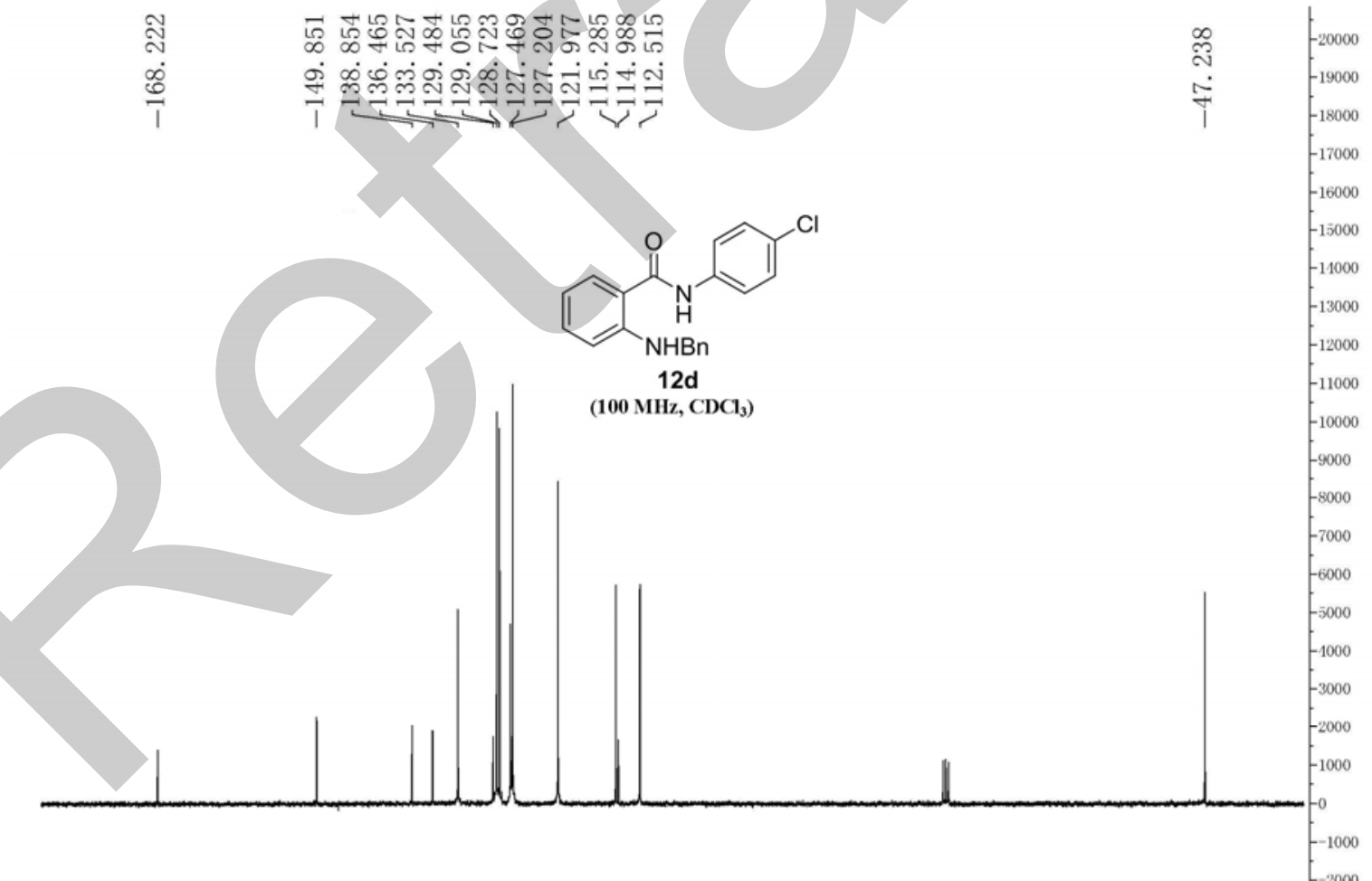

780
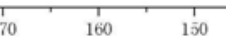

${ }^{1} 140$

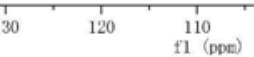

100 

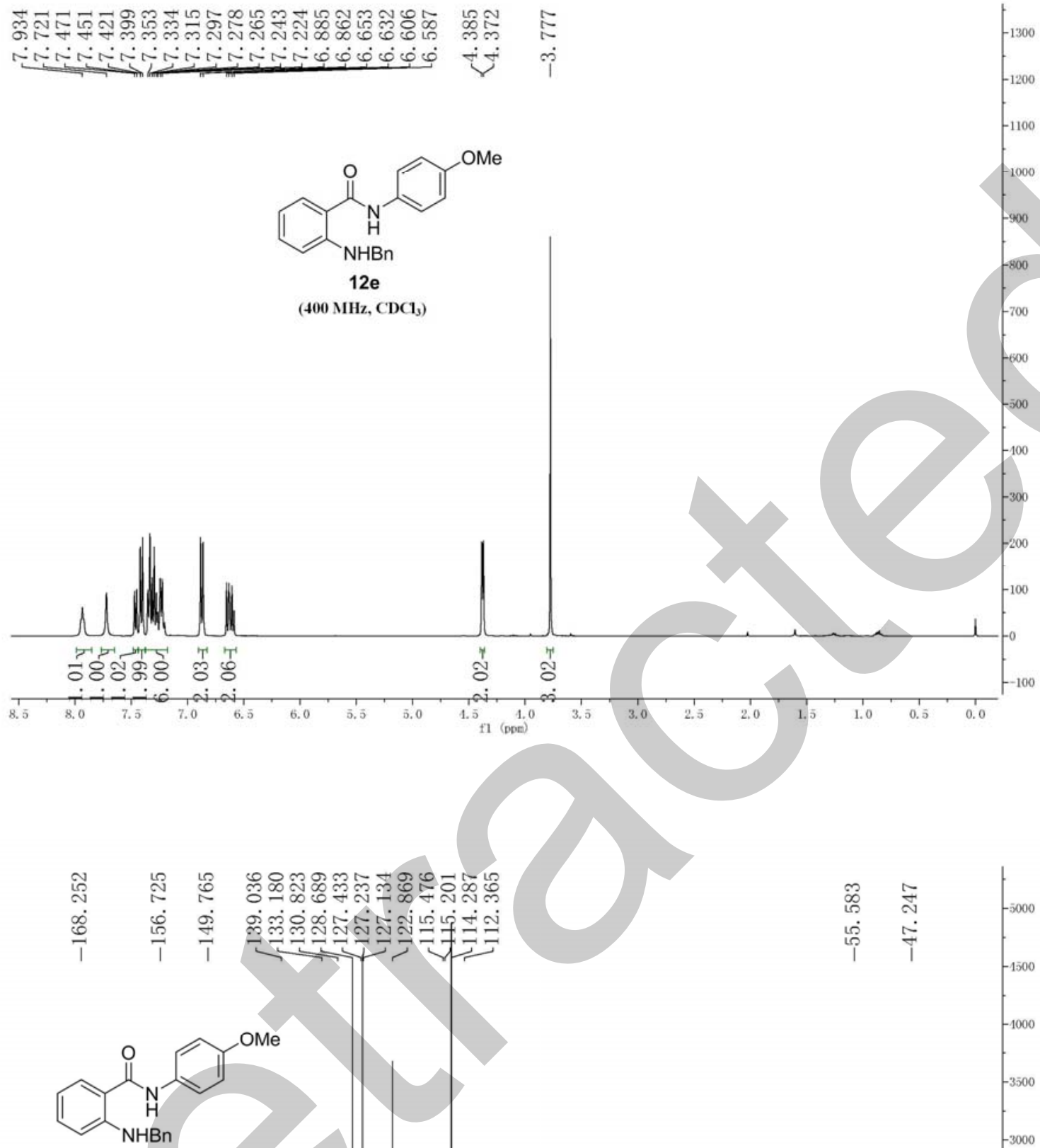

$12 \mathrm{e}$

(100 $\left.\mathrm{MHz}, \mathrm{CDC}_{3}\right)$
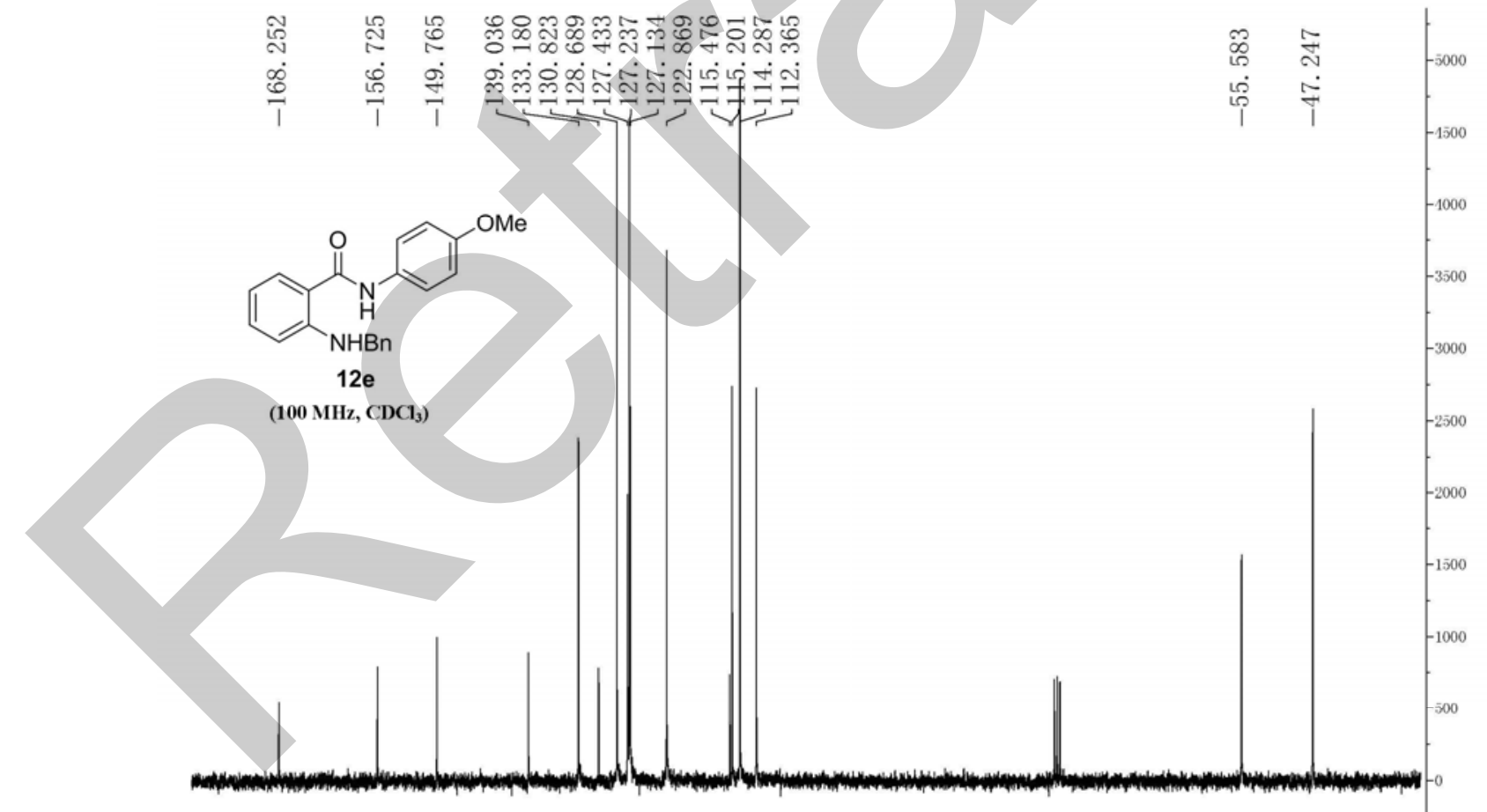

${ }_{170}^{1} \cdot{ }_{160}^{1} \cdot \frac{1}{150}$
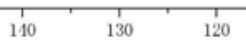

${ }_{11}^{10}$ (ppm) 


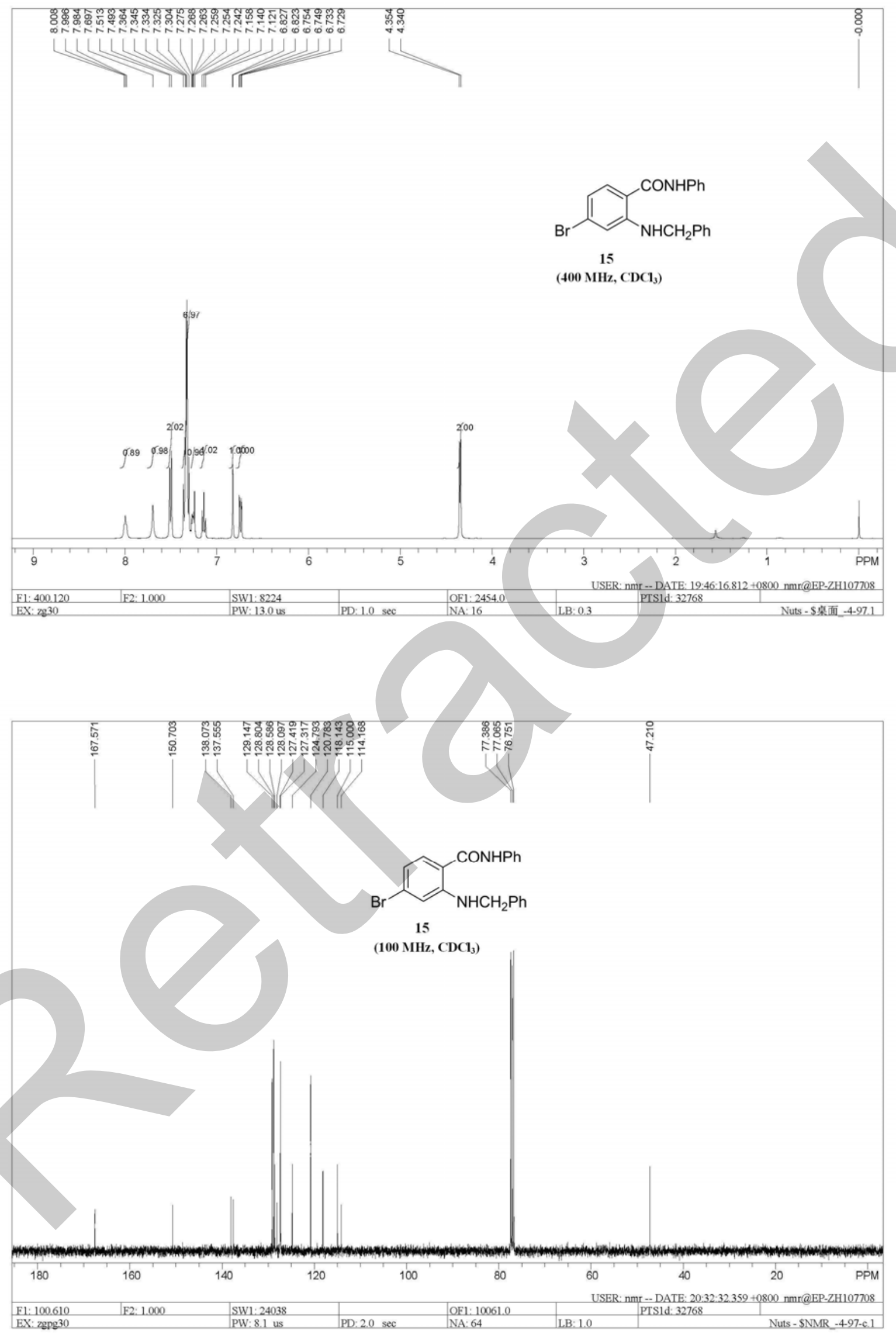

